\title{
PROPRIÉTÉS MAGNÉTIQUES DE L'OXYDE FERRIQUE RHOMBOÉDRIQUE $\left(\mathrm{Fe}_{2} \mathrm{O}_{3} \alpha\right)$
}

\author{
Par Raymond CHEvallieR, \\ Professeur à la Faculté des Sciences de Nancy.
}

Sommaire. - Les propriétés magnétiques essentielles de $\mathrm{Fe}_{2} \mathrm{O}_{3} \alpha$ peuvent se résumer comme il suit : elles sont la superposition d'un paramagnétisme de base variant avec la température suivant la loi de K. Endo,

$$
\left.\begin{array}{rl}
\chi(T+3727) & =0,085 \\
\%(200 \mathrm{C} & =20.10^{-6}
\end{array}\right\}
$$

et d'un ferromagnétisme d'intensité variable, qui disparaît à $\theta=675^{\circ} \mathrm{C}$. En ce même point $\theta$, se produisent simultanément des discontinuités du coefficient de dilatation et de la chaleur spécifique. Cette température est donc un point de transition d'une transformation de second ordre. Au-dessus de $\theta$ l'oxyde est paramagnétique pur; au-dessous paramagnétisme et ferromagnétisme sont superposés. Précisons leur caractère.

Jans les champs très élevés, où l'aimantation est réversible, la superposition s'exprime par la loi simple

$$
\sigma=\sigma_{0}+\chi H \text {. }
$$

où $\sigma_{0}$ est l'aimantation spontanée ferromagnétique et $\chi H$ la contribution du paramagnétisme de Endo. L'aimantation spontanée dépend de la préparation et de l'histoire de l'oxyde; elle ne dépasse jamais i U. E. M. On sait, par exemple, préparer des oxydes de ferromagnétisme inappréciable à la température ordinaire $\left(\sigma_{0}=0\right)$ et qui sont purement paramagnétiques de coefficient voisin de $20.10^{-6}$. Mais tous deviennent ferromagnétiques par recuit à haute température et leur paramagnétisme pur est exceptionnel.

Pour les oxydes courants soumis à des champs moyens, l'aimantation comporte une partie irréversible; les trajets d'aller et retour ne sont plus superposés; l'hystérèse peut être considérable et le champ coercitif atteint parfois 3 ooo gauss. Pourtant, le paramagnétisme de Endo continue à se manifester par la valeur du coefficient d'aimantation réversible dans les champs supérieurs à quelques milliers de gauss. Ce coefficient est, en effet, constant et indépendant de l'origine et de l'histoire de l'échantillon. Sa valeur $\chi=20.1^{-6}$ est identique à la susceptibilité paramagnétique, parce que la susceptibilité réversible ferromagnétique tend vers zéro dans les champs de cet ordre.

Les propriétés magnétiques des monocristaux de $\mathrm{Fe}_{2} \mathrm{O}_{3} \alpha$ apportent quelques données nouvelles. Ils sont rhomboédriques, paramagnétiques suivant l'axe ternaire, isotropes et ferromagnétiques dans le plan normal à cet axe. On retrouve comme coefficient du paramagnétisme suivant l'axe, la valeur habituelle $\chi=20.10^{-6}$ et normalement à l'axe la loi (2) avec une aimantation spontanée de l'ordre de o,5 U. E. M.

Si l'on pulvérise l'un de ces cristaux et qu'on fractionne les grains de façon à grouper ensemble les éléments monocristallins de même taille (diamètre $d$ ), on constate que les propriétés d'une poudre dépendent de $d$. La loi d'aimantation dans les champs forts reste la loi (2) avec $\chi=20$. $10^{-6}$, mais l'aimantation spontanée de l'ordre de 0,4 pour les gros grains, commence à diminuer rapidement autour de $d=$ г оo $\mu$ pour devenir insensible autour de $d=$ I $\mu$. Il paraît difficile d'échapper à la conclusion que le ferromagnétisme ne se manifeste dans le pilan normal à l'axe que si les dimensions du cristal dans ce plan sont suffisantes. Un cristal de i $\mu$ environ dans toutes les directions serait isotrope et présenterait le paramagnétisme de base à l'état pur. Ces propriétés très nettes des poudres de monocristaux n'ont pas encore permis d'interpréter les caractères si variables du ferromagnétisme des poudres de $\mathrm{Fe}_{2} \mathrm{O}_{3} \alpha$ d'origine chimique.

Une dernière question se pose : devons-nous attribuer le paramagnétisme de base à $\mathrm{Fe}_{2} \mathrm{O}_{3} \alpha$ et le ferromagnétisme superposé, qui reste toujours faible, à une impureté étrangère comme la magnétite rhomboédrique de L. Néel; ou bien devons-nous admettre que l'oxyde de composition stœchiométrique $\mathrm{Fe}_{2} \mathrm{O}_{3}$ présenterait déjà un ferromagnétisme spécifique ? Il est difficile d'être catégorique sur ce point. Mais il nous semble que les faits présentés dans cette étude sont plus favorables à la seconde hypothèse. Le déficit ou l'excès d'oxygène dans le réseau de $\mathrm{Fe}_{2} \mathrm{O}_{3}$ peuvent modifier profondément son ferromagnétisme, mais ce ferromagnétisme même préexiste à la modification du réseaul.

1. Polymorphisme de l'oxyde ferrique. L'oxyde ferrique $\mathrm{Fe}_{2} \mathrm{O}_{3}$ est une substance polymorphe dont on a signalé quatre formes cristallines de propriétés magnétiques variées :
La première est rhomboédrique du type corindon. Elle est, en général, très faiblement ferromagnétique. C'est la forme la plus fréquente et la plus stable, celle que présente l'espèce minéralogique hématite 
ou fer oligiste. Par abréviation on la désigne couramment par le symbole $\mathrm{Fe}_{2} \mathrm{O}_{3} \alpha$.

La seconde est cubique du type spinelle. C'est un ferromagnétique fort comparable à la magnétite. Elle est instable; quand on la chauffe, elle se transforme irréversiblement en $\mathrm{Fe}_{2} \mathrm{O}_{3} \alpha$, mais à des températures variables suivant les échantillons. On la note $\mathrm{Fe}_{2} \mathrm{O}_{3} \gamma$.

La troisième est de nouveau rhomboédrique; ses paramètres sont très voisins de ceux de $\mathrm{Fe}_{2} \mathrm{O}_{3} \alpha$. Mais contrairement à cette espèce, c'est un ferromagnétique fort qui possède un point de Curie vers $220^{\circ} \mathrm{C}$. Ce composé est instable; par recuit prolongé au-dessus de $700^{\circ} \mathrm{C}$, il se transforme irréversiblement en $\mathrm{Fe}_{2} \mathrm{O}_{3} \alpha$. Il provient de la décomposition spontanée vers $650^{\circ} \mathrm{C}$ du ferrite de glucinium $\mathrm{Fe}_{2} \mathrm{O}_{3}, 4 \mathrm{GlO}$ [23]. L'existence de cette forme est contestée par A. Michel [33] qui la considère comme un mélange des $\mathrm{Fe}_{2} \mathrm{O}_{3} \alpha$ et $\gamma$.

La dernière forme semble hexagonale [34]. Elle est ferromagnétique, mais très instable, car elle perd déjà son ferromagnétisme à $\mathrm{IOo}^{\circ} \mathrm{C}$. On l'obtient en oxydant l'hydrate ferreux par l'eau oxygénée en milieu alcalin [16]. On a proposé de l'appeler $\mathrm{Fe}_{2} \mathrm{O}_{3}$ う [34].

Le présent Mémoire est consacré à l'étude des propriétés magnétiques de la forme rhomboédrique stable. Étant donnée la complexité des phénomènes d'aimantation présentés par $\mathrm{Fe}_{2} \mathrm{O}_{3} \alpha$ et l'absence de principes indiscutables groupant l'ensemble des propriétés, nous sommes obligés de consacrer une part importante de ce travail à un exposé purement descriptif. Les faits principaux y paraîtront souvent indépendants, mais il est nécessaire de les dégager de toute théorie pour pouvoir, plus tard, en chercher une explication générale.

Il semble, a priori, naturel d'interpréter les propriétés des poudres d'oxyde ferrique par celles des monocristaux et d'exposer tout d'abord les propriétés de ces derniers. Nous ne suivrons pas cet ordre logique pour les deux raisons suivantes :

a. L'oxyde ferrique présentant les propriétés magnétiques les plus simples n'est pas le monocristal, mais l'oxyde artificiel provenant de la déshydratation de la gœthite.

$b$. On ne parvient pas à expliquer les propriétés des poudres d'origine chimique par celles des monocristaux.

Insistons sur ce dernier point. Dans une poudre de grains monocristallins (Cf. $\S 10)$ de même dimension moyenne, les propriétés magnétiques sont à peu près définies par la taille des grains, mais il est essentiel pour cela qu'un même réseau occupe le volume entier du grain; on ne pourra l'affirmer en toute certitude que si le grain provient de la fragmentation d'un monocristal. Quand un oxyde ferrique artificiel se forme par voie chimique et que les molécules $\mathrm{Fe}_{2} \mathrm{O}_{3}$ s'associent progressivement à basse température, chaque grain peut être un groupe mâclé de monocristaux plus ou moins imparfaits et lacunaires; les tensions internes sont inconnues; des gaz adsorbés ou dissous $\left(\mathrm{O}_{2}, \mathrm{H}_{2} \mathrm{O}, \mathrm{NO}_{2}, \mathrm{SO}_{2}, \ldots\right)$ peuvent s'insérer dans le réseau, bref chacun de ces grains à structure indéterminée ne saurait être comparé à un monocristal. Sa taille n'est pas une variable essentielle et ses propriétés magnétiques sont imprévisibles. Il n'y a donc pas lieu de s'étonner qu'à taille de grains à peu près identique, les propriétés des poudres de $\mathrm{Fe}_{2} \mathrm{O}_{3}$ obtenues par voie chimique et celles des poudres provenant de la fragmentation d'un monocristal soient profondément différentes; nous les exposerons indépendamment.

Une première partie traitera des poudres à grains polycristallins, une seconde de celles à grains monocristallins; dans une dernière partie nous grouperons les essais d'interprétation.

2. Notations. - Nous aurons à utiliser couramment les constantes magnétiques habituelles des substances ferromagnétiques. Précisons, dès maintenant les notations adoptées :

$H, h, \quad$ champ magnétique appliqué en gauss. Dans nos expériences, le champ maximum fut de 6500 gauss;

$\mathscr{J}$ intensité d'aimantation (rapportée à $\left.\mathrm{I} \mathrm{cm}^{3}\right)$;

$\sigma, \quad$ aimantation par gramme de substance magnétique (aimantation spécifique);

$\% \quad$ coefficient d'aimantation ou susceptibilité spécifique;

\%, coefficient d'aimantation initial;

$\chi_{1}, \quad$ coefficient d'aimantation vrai $\frac{d \sigma}{d H}$ pour le champ maximum $6500 \mathrm{~g}\left(\chi^{\prime}\right.$, courbe d'aller, $\chi^{\prime \prime}$ courbe de retour);

$\%, \quad$ coefficient d'aimantation réversible;

$\sigma_{m}, \quad$ aimantation atteinte dans le champ maximum de 65 oo g;

$\sigma ., \quad$ aimantation rémanente pour le champ maximum;

$H_{\text {, }} \quad$ champ coercitif relatif au champ maximum;

$d$, diamètre moyen des grains de la poudre, en microns;

$\Theta^{\circ} \mathrm{K}, 0^{\circ} \mathrm{C}$, points de Curie dans l'échelle absolue et dans l'échelle centigrade.

On remarquera que dans l'aimantation de $\mathrm{Fe}_{2} \mathrm{O}_{3} \alpha$, le champ démagnétisant est toujours négligeable devant le champ appliqué.

D'autre part, $\sigma_{2}$ et $H_{c}$ dépendent du champ maximum, qui est relativement faible. Ces grandeurs croîtraient certainement si l'on pouvait atteindre 30 ooo gauss comme dans les expériences de Mlle J. Roquet [38]. 
I. - Poudres de $\mathrm{Fe}_{2} \mathrm{O}_{3} \alpha$, à grains polycristallins.

3. L'oxyde ferrique rhomboédrique paramagnétique. -- Il existe deux hydrates ferriques cristallisés de constitution $\mathrm{Fe}_{2} \mathrm{O}_{3} \mathrm{H}_{2} \mathrm{O}$, tous les deux orthorhombiques, existant aussi bien comme espèces naturelles qu'en microcristaux synthétiques. La gœthite, de couleur jaune en poudre fine, fournit, par déshydratation $\mathrm{Fe}_{2} \mathrm{O}_{3} \alpha$, la lépidocrocite qui est rouge $\mathrm{Fe}_{2} \mathrm{O}_{3} \gamma$.

Nous nous occuperons ici de l'oxyde dérivé de la gœthite qui représente la variété de $\mathrm{Fe}_{2} \mathrm{O}_{3} \alpha$ de propriétés magnétiques les plus simples.

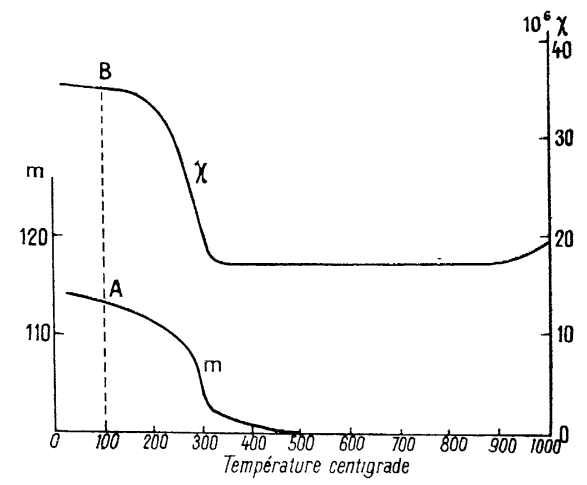

Fig. I. - Déshydratation de la gœthite $\left(\mathrm{Fe}_{2} \mathrm{O}_{3}, \mathrm{H}_{2} \mathrm{O}\right)$ et recuit à l'air de $\mathrm{Fe}_{2} \mathrm{O}_{3} \alpha$ dérivé.

$m$, masse de substance après recuit à la température considérée, pour roo mg de $\mathrm{Fe}_{2} \mathrm{O}_{3}$ final; $\%$, coefficient d'aimantation de la dite substance.

La figure I représente la marche de la déshydratation et le recuit de l'oxyde dérivé. La substance est chauffée progressivement de 50 en $50^{\circ}$ dans l'intervalle $100-500^{\circ} \mathrm{C}$ et de 100 en $100^{\circ}$ entre 500 et $\mathrm{IO00}^{\circ} \mathrm{C}$. A chaque température ( 100 , i5o, etc.) la substance est maintenue $30 \mathrm{mn}$, refroidie, pesée et mesurée magnétiquement. On désigne par $m$ sa masse rapportée à $100 \mathrm{mg}$ de $\mathrm{Fe}_{2} \mathrm{O}_{3}$ final et par $\chi$ son coefficient d'aimantation mesuré à la balance de Curie. A chaque température correspond donc un couple de points A, B. Le lieu de A définit la déshydratation, le lieu de $B$ la variation de $\chi$ concomitante. Le phénomène magnétique suit exactement la déshydratation, comportement très simple èt d'ailleurs exceptionnel qu'on ne retrouve pas chez les hydroxydes colloïdaux. A $400^{\circ} \mathrm{C}$, on voit que toute l'eau est pratiquement partie; $\%$ a atteint sa valeur minimum $18.1^{-6}$ et ne varie plus par recuit jusqu'à $1000^{\circ} \mathrm{C}$. Choisissons un échantillon d'oxyde, par exemple celui recuit à $500^{\circ} \mathrm{C}$ et précisons ses propriétés.

I $^{\circ}$ C'est une poudre rouge extrêmement fine dont nous avons tout d'abord déterminé la loi d'aiman- tation [36]. Elle est rigoureusement rectiligne (fig. 2); à la précision près de nos mesures les points d'aller et de retour sont sur la même droite. Le coeffieient d'aimantation correspondant est de l'ordre de $\%=18.10^{-6}$.

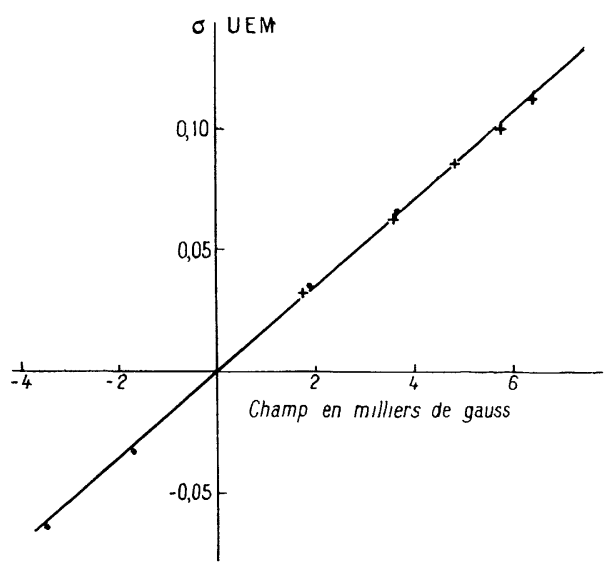

Fig. 2. - Loi d'aimantation de $\mathrm{Fe}_{2} \mathrm{O}_{3} \propto$ paramagnétique.

+ mesures par champs croissants.

- mesures par champs décroissants.

$2^{0}$ Par une expérience spéciale avec une balance de torsion sensible, nous avons vérifié que l'oxyde soumis à un champ de 6500 gauss ne conserve aucun moment magnétique permanent.

$3^{\circ}$ L'oxyde est placé dans un champ magnétique d'une centaine de gauss et l'on fait croître sa température de 20 à $750^{\circ} \mathrm{C}$. Son moment magnétique reste constant, comme le montre la figure $3 a$ qui

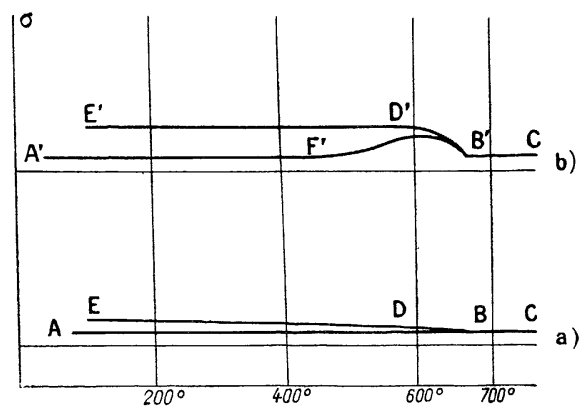

Fig. 3. - Courbes thermomagnétiques de $\mathrm{Fe}_{2} \mathrm{O}_{3} \alpha$. a. Non recuit. Paramagnétisme.

b. Recuit à $1000^{\circ} \mathrm{C}$. Le ferromagnétisme apparaît.

reproduit la courbe thermomagnétique enregistrée. Sur le trajet d'aller $\mathrm{ABC}$ le passage par $675^{\circ}$ n'est accusé par aucune inflexion. Une série de mesures effectuées de - $196^{\circ}$ (azote liquide) à $20^{\circ} \mathrm{C}$ n'a montré de même aucune variation du moment magnétique. 
$t^{\circ}$ Par refroidissement dans le champ constant ( $f i g .3 a$, trajet $\mathrm{CBDE}$ ) la courbe de retour se détache en $\mathrm{B}$ de la courbe d'aller et le moment magnétique croît jusqu'à la température ordinaire. Nous avons vérifié qu'en $\mathrm{E}$, la substance avait acquis un moment permanent. L'oxyde se comporte donc comme un paramagnétique à susceptibilité indépendante de la température tant qu'il n'atteint pas $700^{\circ}$ environ. Au-dessus un ferromagnétisme faible commence à apparaître et devient de plus en plus net quand on élève la température de recuit et qu'on augmente la durée de ce recuit.

$5^{\circ}$ On a chauffé l'oxyde à I $^{\circ} 0^{\circ}$ pendant i h dans l'oxygène pour éviter toute réduction possible. La loi d'aimantation est restée rectiligne, et l'hystérèse est restée inappréciable. Le coefficient d'aimantation a peu varié : $\mathrm{ro}^{6} \chi=19$. Mais la courbe thermomagnétique présente maintenant une particularité caractéristique des formes microcristallines de $\mathrm{Fe}_{2} \mathrm{O}_{3} \alpha$ $(f i g .3 b)$. Vers $500^{\circ}\left(F^{\prime}\right)$ le moment croît, passe par un maximum et subit une chute brusque qui le ramène à sa valeur de départ vers $675^{\circ}$. Au retour, le moment reste constant après la remontée $675^{\circ}$ et nous avons vérifié que la substance possède en $\mathrm{E}^{\prime}$ un moment permanent. Cette discontinuité du moment en $\mathrm{B}^{\prime}$ est un véritable point de Curie. L'oxyde $\mathrm{Fe}_{2} \mathrm{O}_{3} \alpha$ par recuit est devenu ferromagnétique et cette température $0=675^{\circ} \mathrm{C}$ est caractéristique de cette espèce.

4. Le ferromagnétisme de $\mathrm{Fe}_{2} \mathrm{O}_{3} \alpha$. - Il est rare que cette phase paramagnétique puisse être observée, soit que la température de formation de l'oxyde soit trop élevée, soit qu'une structure secondaire instable précède la formation de $\mathrm{Fe}_{2} \mathrm{O}_{3} \alpha$ normal.

Le ferromagnétisme se manifeste alors par une courbe de première aimantation non rectiligne, tantôt tournant sa concavité vers le bas pour les champs élevés ( $i g$. 8, 9, I o, I I) et tantôt tournant cette concavité vers le haut dans tout le domaine des champs parcourus (fig. 4). La courbe de retour accuse une hystérèse plus ou moins importante. Le ferromagnétisme disparaît à la température fixe de $675^{\circ} \mathrm{C}$ pour tous les échantillons. C'est C. Berndt [5] qui a tracé le premier cycle d'hystérèse. Honda et Soné [6] ont rencontré la discontinuité de $675^{\circ}$, mais l'ont attribuée à une impureté; c'est H. Forestier [17] qui a montré qu'elle était spécifique de $\mathrm{Fe}_{2} \mathrm{O}_{3} \alpha$.

Nous montrerons d'abord la complexité des phénomènes sur un exemple typique que nous traiterons en détail. Nous situerons ensuite les divers cas rencontrés par rapport à cet exemple.

${ }_{1}^{0}$ Le nitrate ferrique cristallisé, que l'on peut avoir très pur et absolument exempt de fer ferreux, fond à basse température dans son eau de cristallisation, perd de l'eau et de l'acide azotique et donne avant $300^{\circ}$ un oxyde ferrique dont la struc- ture aux rayons X n’a pas encore été précisée, mais qui est probablement la variété rhomboédrique. Cet oxyde forme une masse compacte présentant de belles surfaces réfléchissantes au contact des parois de la coupelle, miroirs comparables à des faces d'hématites (fer spéculaire). Cette masse pulvérisée, tamisée, broyée et lévigée fournit des fractions à grains moyennement identiques qui ont toutes donné la même courbe d'aimantation (fig. \&,

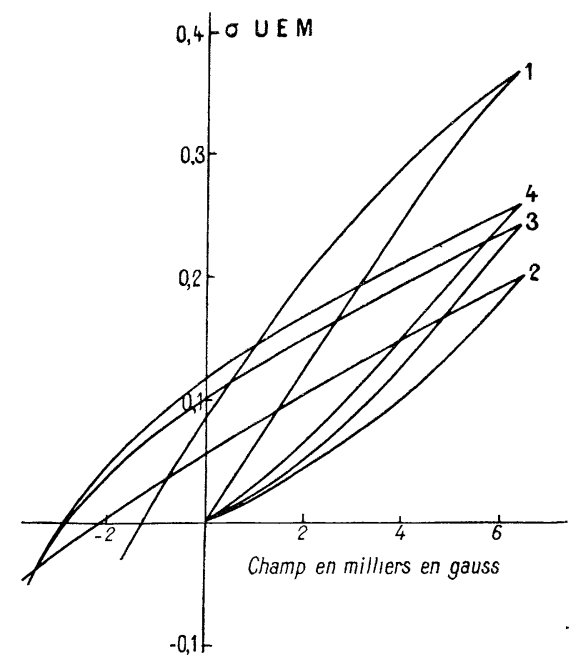

Fig. 4. - Oxyde ferrique dérivé du nitrate. Courbes d'aimantation. Influence du recuit dans l'air à $1000^{\circ} \mathrm{C}$

courbe 1). Malgré la température très basse de préparation, l'oxyde présente une forte hystérèse et, en particulier, un champ coercitif de i 250 gauss environ. Le broyage est sans influence, parce qu'il brise les groupes de cristaux, mais n'atteint pas les cristaux eux-mêmes.

$2^{\circ}$ Un recuit par températures croissantes diminue tout d'abord les propriétés magnétiques comme L. Blanc [13, 15] l'a remarqué autrefois, mais tend à augmenter le champ coercitif. Pour une température non précisée, la courbe d'aimantation devient stationnaire, après quoi les propriétés magnétiques augmentent lentement par recuit et semblent tendre vers une limite. Le champ coercitif va constamment en croissant. Le tableau ci-dessous précise cette évolution.

$3^{\circ}$ Nous avons tracé, sur la figure 4 à titre d'exemple, les courbes 2 et 3 correspondant aux oxydes recuits à $1000^{\circ} \mathrm{C}$ respectivement $\mathrm{i} 5 \mathrm{mn}$ et $4 \mathrm{~h}$. Ces courbes sont à peu près semblables comme les courbes intermédiaires non tracées. Le recuit amène un accroissement régulier des ordonnées qui conduit à la courbe limite 3 quand la durée du recuit devient très longue. On remarquera la valeur constante de $\chi_{l}$ sur la courbe de retour à partir de $400^{\circ}$ 
Tableav 1.

$\begin{array}{ccc}\text { Tempér. Durée en mm. } & 10^{\prime} \%_{\nu} \\ 2-300^{\circ} & 60^{\prime} \ldots \ldots & 62 \\ 400 & 60 \ldots \ldots & 53 \\ 600 & 30 \ldots \ldots \ldots & 34 \\ 1000 & 15 \ldots \ldots & 20 \\ 1000 & 60 \ldots \ldots & 20 \\ 1000 & 240 \ldots \ldots \ldots & 20\end{array}$

$\mathrm{ro}^{\mathrm{i}} \chi \imath \neq \#$ 20. Nous reviendrons longuement sur ce point. Nous parlerons plus loin de la courbe 4 .

$4^{\circ}$ Les courbes thermomagnétiques de ces divers oxydes sont tout à fait semblables à celles de la figure 13 . Le maximum est beaucoup plus accusé que sur la figure $3 b$ pour un même champ inducteur; ce phénomène semble lié à l'importance du champ coercitif, comme nous le verrons dans un paragraphe spécial.

Le point de Curie se place toujours à la même température : $\theta=675^{\circ} \mathrm{C}$, qui est une constante de $\mathrm{Fe}_{2} \mathrm{O}_{3} \alpha$.

5. Classification des divers types d'oxydes d'après leur origine et leur histoire. - A la lumière de ces deux exemples, nous allons situer les diverses variétés d'oxydes ferriques rhomboédriques d'après leur mode de préparation.

I $^{0}$ Les sels ferriques oxygénés à acides volatils se décomposent à température convenable en laissant un résidu de $\mathrm{Fe}_{2} \mathrm{O}_{3} \alpha$. Les trois principaux sels à considérer sont le nitrate, le sulfate et l'alun ferriammoniacal. Ces deux derniers sels ont une température de décomposition très supérieure à celle du nitrate et l'oxyde obtenu est automatiquement porté vers $700^{\circ}$ si l'on veut que la décomposition soit complète. Il a des propriétés semblables pour ces deux sels $\left(\sigma_{m} \# 0,35, H_{c}=1000\right.$ à 1500 gauss). Son hystérèse est donc considérable dès sa formation.

Par recuit à $1000^{\circ}$ dans l'air ces constantes évoluent peu contrairement à ce qui se passe pour l'oxyde du nitrate. Au bout de $3 \mathrm{~h}$ on a trouvé ( $H_{c}=\mathrm{I} 800$ gauss). Le champ coercitif augmente toujours par recuit.

$2^{\circ}$ Les hydroxydes colloïdaux précipités des solutions de sels (chlorure, nitrate, sulfate) par les bases $\left(\mathrm{NaOH}, \mathrm{KOH}, \mathrm{NH}_{4} \mathrm{OH}\right)$, puis lavés et séchés, sont complètement déshydratés avant $400^{\circ} \mathrm{C}$; mais l'oxyde obtenu a des propriétés toutes différentes de celui fourni par la gœthite $\left(\mathrm{I}^{6} \chi_{i} \neq 200\right.$ au lieu de 20). L'instabilité de cet oxyde se manifeste par un dégagement de chaleur au cours de sa transformation en $\mathrm{Fe}_{2} \mathrm{O}_{3} \alpha$ et dans les cas favorables par son incandescence. Un léger recuit vers 700 à $800^{\circ}$ a conduit aux constantes suivantes pour un échan-

\begin{tabular}{|c|c|c|c|}
\hline$\sigma_{m}$ & $\sigma_{,}$. & $H_{c}$ & Observations. \\
\hline 0,$3 ; 2$ & 0,085 & 1270 & Courbe $1(\mathrm{fig} \cdot 4)$ \\
\hline$o, 300$ & $o, 093$ & 1300 & \\
\hline 0,257 & $o, 069$ & I575 & \\
\hline 0,202 & 0,052 & 2150 & Courbe $2(\pi g \cdot 4)$ \\
\hline 0,226 & 0,079 & 2500 & \\
\hline$o, 239$ & 0,094 & 2700 & Courbe $3(\pi$ \\
\hline
\end{tabular}

tillon particulier :

$$
\begin{gathered}
\mathrm{r}^{6} \chi_{i}=19,6, \quad \mathrm{1}^{6} \chi_{l}=\mathrm{1} 8,5, \quad \sigma_{m}=0,126, \\
\sigma_{r}=0,005, \quad H_{c}=230 .
\end{gathered}
$$

L'hystérèse est très légère. Cet oxyde s'apparente à la variété de la gœthite et s'écarte beaucoup des variétés dérivées des sels oxygénés.

Un recuit prolongé à $1000-1100^{\circ}$ conduit à des propriétés complexes insuffisamment étudiées, dont les ions alcalins restant peuvent être responsables.

$3^{\circ}$ Les variétés de l'oxyde ferrique cubique se transforment à des températures variables en $\mathrm{Fe}_{2} \mathrm{O}_{3} \alpha$. Mais lorsque ces oxydes sont dérivés de substances ayant séjourné longuement avec des bases, il apparaît par recuit vers $1000^{\circ} \mathrm{C}$ des ferromagnétiques nouveaux insuffisamment étudiés. Les oxydes $\mathrm{Fe}_{2} \mathrm{O}_{3} \alpha$ dérivés sont donc à écarter.

$4^{\circ}$ Enfin des sels ferreux ( $\mathrm{SO}_{4} \mathrm{Fe}$ par exemple) par calcination à l'air ou dans l'oxygène, fournissent de l'oxyde rhomboédrique. Pour éviter la possibilité d'une trace de magnétite troublant par son ferromagnétisme les propriétés de $\mathrm{Fe}_{2} \mathrm{O}_{3} \alpha$, nous écartons les oxydes de cette origine.

Il résulte de cette discussion que pour étudier les propriétés de $\mathrm{Fe}_{2} \mathrm{O}_{3} \alpha$ sans qu'elles soient compliquées par les propriétés d'impuretés additionnelles, il faut avoir recours à l'oxyde dérivé d'une gœthite pure que l'on déshydrate ou d'un sel oxygéné que l'on décompose.

6. Susceptibilité réversible [41]. - De la complexité des courbes d'aimantation de la figure 4 et des nombreuses courbes semblables, ressort un fait constant qui doit attirer notre attention :

Le coefficient vrai d'aimantation à 6500 gauss sur la courbe de retour est invariable et voisin de $20.10^{-6}$.

Cette conclusion se dégage du Tableau I, où le premier oxyde de structure douteuse est à écarter; d'autre part, on pourra la vérifier sur toutes les courbes de ce Mémoire.

Ce résultat doit se rattacher à une propriété générale de $\mathrm{Fe}_{2} \mathrm{O}_{3} \alpha$ que nous allons chercher à préciser.

ro Utilisant l'oxyde ferrique dérivé du nitrate et 
recuit $4 \mathrm{~h}$ dans l'air à $1000^{\circ} \mathrm{C}$ (fig. 4, courbe 3), nous avons construit les courbes de retour pour divers champs maximum.

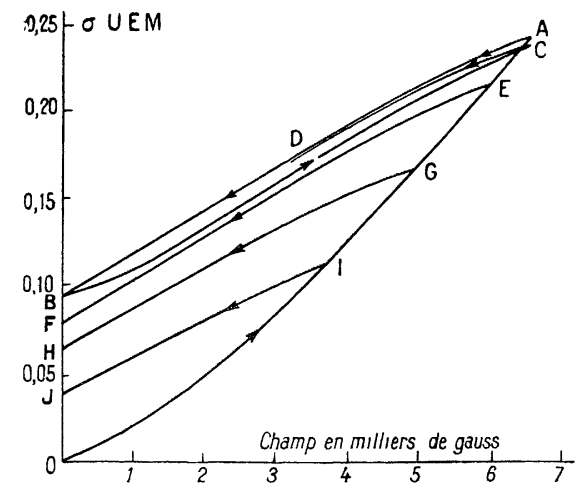

Fig. 5. - Courbes d'aimantation de $\mathrm{Fe}_{2} \mathrm{O}_{3} \alpha$. Parallélisme des trajets de retour.

Les résultats sont représentés figure $\tilde{\jmath}$. Voici la suite des opérations :

La poudre désaimantée est portée dans un champ de 6500 gauss (point $\mathrm{A}$ ), ramenée à zéro, portée à 6500 gauss, etc. On décrit le trajet ABCD que l'on détermine par points. Le cycle se ferme pratiquement en $D$. Il est très aplati et presque rectiligne d'inclinaison 20.1 $\mathrm{1}^{-6}$. On peut dire que le trajet $\mathrm{AB}$ est quasi réversible, aussi ne l'a-t-on parcouru qu'une fois pour les champs suivants. La poudre est agitée de façon à revenir à un moment nul, portée dans un champ plus faible $E$ et ramenée à zéro, trajet $\mathrm{EF}$, de même pour les champs successifs $G$, I. Il est frappant que les trajets de retour $A B$, $\mathrm{EF}, \mathrm{GH}, \mathrm{IJ}$ soient presque rectilignes et parallèles de direction moyenne $\chi=20.10^{-6}$. Ce résultat suggère que le coefficient d'aimantation réversible doit être constant dans tout le domaine et précisément égal à $20 \cdot 10^{-6}$.

$2^{0}$ Rappelons la définition de ce coefficient (fig. 6).

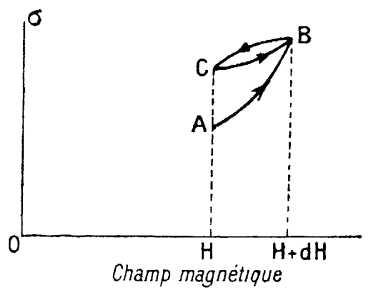

Fig. 6. - Définition du coefficient d'aimantation réversible.

Quand le champ H croît infiniment peu, le point $(\sigma, H)$ passe par exemple de $\mathrm{A}$ en $\mathrm{B}$. Si l'on ramène le champ à sa valeur initiale et qu'on le fasse osciller entre $H$ et $H+\mathrm{d} H$, le point représentatif finit par décrire un cycle fermé BC infiniment plat dont l'inclinaison $\chi_{\sigma}=\frac{\mathrm{d} \sigma}{\mathrm{d} \boldsymbol{H}}$ est, par définition, le coefficient d'aimantation réversible.

Étant donnée la petitesse des aimantations à mesurer dans le cas de $\mathrm{Fe}_{2} \mathrm{O}_{3} \alpha$, nous avons dû prendre comme intervalle $\mathrm{d} H$ un accroissement de rooo gauss et nous avons déterminé $\chi_{r}$. sur la première ascendante pour trois substances :

a. $\mathrm{Fe}_{2} \mathrm{O}_{3} \alpha$ dérivé du nitrate et recuit $4_{4} \mathrm{~h}$ à l'air à $1000^{\circ}$;

b. $\mathrm{Fe}_{2} \mathrm{O}_{3} \alpha$ dérivé du sulfate et recuit $4 \mathrm{~h}$ à l'air à $1000^{\circ}$;

c. $\mathrm{Fe}_{2} \mathrm{O}_{3}$ provenant de la décomposition du nitrate $\left(200-300^{\circ}\right)$.

Les résultats sont représentés sur la figure 7 .

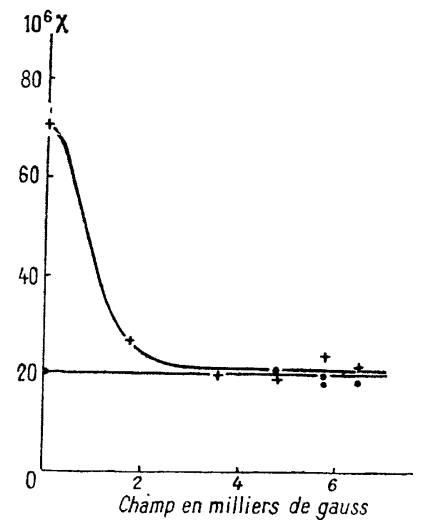

Fig. 7. - Constance du coefficient d'aimantation réversible.

- $\mathrm{Fe}_{3} \mathrm{O}_{3} \propto$ dérivé du sulfate (2 séries de mesures).

$\times \mathrm{Fe}_{2} \mathrm{O}_{3} \propto$ dérivé du nitrate ( $\mathrm{I}$ série de mesures), recuits l'un et l'autre à $1000^{\circ} \mathrm{C}$.

Les mesures sont très imprécises, car elles portent sur des différences fort petites. Une conclusion certaine se dégage pourtant des nombres obtenus.

Pour l'oxyde rhomboédrique dérivé du nitrate ou du sulfate, $\chi_{r}$. tend dans les champs élevés vers la valeur constante $20 . \mathrm{IO}^{-6}$; pour un oxyde instable dérivé du nitrate, nous avons trouvé, par exemple, un coefficient de l'ordre de $40.10^{-6}$, ce qui montre bien que la valeur $20.10^{-6}$ de $\chi_{r}$ est spécifique de la forme rhomboédrique. Nous formulerons donc le principe suivant valable pour toutes nos mesures :

Le coefficient d'aimantation réversible de l'oxyde ferrique rhomboédrique a la valeur constante $\psi_{r}=20 . \mathrm{I}^{- \text {;; }}$ dès que le champ atteint quelques milliers de gauss. Cette valeur ne dépend ni de l'origine de l'oxyde, ni de son histoire.

Tout accroissement d'aimantation $\mathrm{d} \sigma$ correspondant à un accroissement de champ $\mathrm{d} H$ comporte donc une partie réversible $\gamma_{r} \mathrm{~d} H$ qui est la même pour tous les oxydes et une partie irréversible qui 
varie d'un oxyde à l'autre et dépend des structures secondaires. Nous verrons que pour les poudres à grains monocristallins cette partie irréversible est très faible dans les champs élevés : la courbe d'aimantation rectiligne et réversible a la pente prévue $y_{r}=20 \cdot \mathrm{IO}^{-6}$. Pour les oxydes à grains polycristallins, la partie irréversible peut être fort importante sur la première ascendante, mais elle est toujours très petite sur la courbe de retour, d'où la pente observée voisine de $\%$.

7. Présence d'ions ferreux dans le réseau de $\mathrm{Fe}_{2} \mathrm{O}_{3} \alpha$. - Doit-on attribuer le ferromagnétisme de l'oxyde ferrique rhomboédrique à la présence d'ions ferreux dans son réseau ? L. Néel [40] s'appuyant sur les expériences de Snoëk admet cette hypothèse et a construit une théorie attribuant ce ferromagnétisme à une dispersion de magnétite dans $\mathrm{Fe}_{2} \mathrm{O}_{3} \alpha$ pur. Nous allons voir que les travaux de Sosman et Hostetter [8, 9, 10] démontrent la présence presque constante d'ions ferreux dans le réseau et le rôle important qu'ils jouent dans les propriétés magnétiques. En revanche, la conclusion que Snoëk a tirée de ses expériences [28] ne saurait être retenue et il existe certainement d'autres facteurs que les ions ferreux pour contribuer au ferromagnétisme de $\mathrm{Fe}_{2} \mathrm{O}_{3}$.

$1^{\circ}$ Le premier phénomène à considérer dans la formation d'ions ferreux est la tension de dissociation de $\mathrm{Fe}_{2} \mathrm{O}_{3}$. Voici ce qui résulte des expériences de Sosman et Hostetter [8] :

Quand $\mathrm{F}_{2} \mathrm{O}_{3} \alpha$ pur est soumis à une température de ${\mathrm{I} I 00^{\circ}}^{\circ}$, sa tension de dissociation est de l'ordre du millimètre de mercure. Si l'on permet à l'oxygène de se dégager, l'oxyde magnétique libéré ne forme pas une phase nouvelle, mais reste dissous dans $\mathrm{Fe}_{2} \mathrm{O}_{3}$, en sorte que le système est bivariant. A température constante, la tension de dissociation change, on trouve qu'elle décroît rapidement avec la concentration en $\mathrm{Fe}_{3} \mathrm{O}_{4}$, qu'elle tombe, par exemple, à $0,2 \mathrm{~mm}$ quand la concentration est de 5 pour ioo. Pour $\mathrm{Fe}_{2} \mathrm{O}_{3} \alpha$ pur, la courbe de tension quitte l'axe presque verticalement et sa valeur sur l'axe est très mal connue, mais il semble bien qu'à I I00 elle ne saurait dépasser quelques millimètres. Dès lors, comment pourrait-il y avoir formation de magnétite dans l'oxyde pur chauffé à l'air à $\mathrm{I}_{100^{\circ}}$, puisque la tension de l'oxygène dans l'air est i $50 \mathrm{~mm}$. Il faut nécessairement qu'intervienne un réducteur.

$2^{\circ}$ Un second facteur a été étudié par Sosman et Hostetter dans une deuxième série d'expériences [9]. Une certaine masse de $\mathrm{Fe}_{2} \mathrm{O}_{3}$ chauffée alternativement dans l'air, puis dans l'oxygène

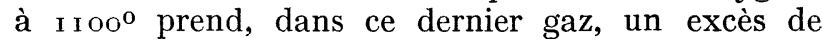
masse de $\mathrm{I} / \mathrm{IO}^{\circ} \mathrm{Ooo}^{\mathrm{e}}$ par rapport à la masse prise dans l'air. Il est probable que pour une pression d'oxygène suffisante $\mathrm{Fe}_{2} \mathrm{O}_{3}$ adsorbe $\mathrm{O}_{2}$, phénomène complémentaire de la dissociation qui consiste en départ d'oxygène quand la pression est assez faible. $\mathrm{La}$ constitution stœchiométrique $\mathrm{Fe}_{2} \mathrm{O}_{3}$ ne serait pratiquement jamais réalisée. Il y aurait toujours déficit ou excès d'oxygène suivant la pression de $\mathrm{O}_{2}$ et la température. Les auteurs concluent : " $\mathrm{La}$ grande facilité de dissociation des premières portions de $\mathrm{Fe}_{2} \mathrm{O}_{3}$ explique ce fait que sur 75 échantillons naturels ou artificiels analysés dans ce laboratoire, un seul n'a pas présenté une quantité mesurable de fer ferreux." "

Comme au paragraphe précédent, nous remarquerons que la présence de $\mathrm{FeO}$ s'accorde mal avec la tension de dissociation. C'est un excès d'oxygène qu'on devrait trouver en l'absence de réducteur.

$3^{\circ}$ Puisque la présence de fer ferreux est expérimentalement certaine, les propriétés magnétiques sont-elles proportionnelles à la concentration en $\mathrm{FeO}$ ? Ce problème fait l'objet d'un troisième Mémoire [10]. Quand la concentration est inférieure à 0 , I pour I 00 de $\mathrm{FeO}$, on remplace le titrage chimique par un titrage électrométrique qui, suivant les auteurs, permet d'apprécier jusqu'à o,o I pour ıoo de $\mathrm{FeO}$. Les mesures magnétiques, effectuées par pesée dans un champ hétérogène, n'ont qu'un sens qualitatif. On trouve que pour des quantités de $\mathrm{FeO}$ supérieures à I pour roo ( 3 pour ioo de $\mathrm{Fe}_{3} \mathrm{O}_{4}$ ) le magnétisme correspond à peu près à $\mathrm{FeO}$ présent, mais au-dessous il est sans rapport avec la concentration en ions ferreux : des oxydes ayant même concentration (o, I pour Ioo de $\mathrm{FeO}$ ) ont fourni des attractions variant dans le rapport de i à ıo.

L'ensemble de ces mesures appelle quelques réserves. La sensibilité et la fidélité supposées par les auteurs semblent grandes. L'interprétation de la deuxième série d'expériences porte sur une différence de masse de $\mathrm{I} / \mathrm{I} O 000^{\mathrm{e}}$ après chauffage à $\mathrm{I} 200^{\circ}$. La possibilité de doser dans $\mathrm{Fe}_{2} \mathrm{O}_{3}$ I/100 de $\mathrm{FeO}$ à quelques centièmes près laisse rêveur. On aimerait à voir ces expériences répétées avec contrôle magnétique rigoureux. En attendant, il faut retenir les conclusions générales du travail : présence fréquente d'ions ferreux (déficit d'oxygène) dans les oxydes recuits à l'air au-dessus de $1000^{\circ}$; excès d'oxygène pour ceux recuits à des températures semblables dans l'oxygène à la pression atmosphérique. Sauf pour les grandes concentrations en $\mathrm{FeO}$ (supérieures à I pour Ioo) les propriétés magnétiques restent imprévisibles. Voyons ce que l'expérience nous apprend à ce sujet.

8. Propriétés magnétiques de $\mathrm{Fe}_{2} \mathrm{O}_{3} \alpha$ recuit dans l'oxygène. - En r 93 I , Hüttig et Kittel [20] ayant chauffé un échantillon de $\mathrm{Fe}_{2} \mathrm{O}_{3}$ dans l'oxygène pendant $4 \mathrm{~h}$, trouvèrent que le coefficient d'aimantation était passé de 22,8.10 $0^{-6}$ à $57,6 \cdot 10^{-6}$. Ils attribuèrent cette croissance à l'oxygène adsorbé. En I 937, Snoëk [28], partant d'un oxyde ferrique de 
provenance et d'histoire inconnues, mesure son intensité d'aimantation à saturation $\mathfrak{J}_{m}=4,65(\sigma=0,90$ avec une densité de $\tilde{5}, 2)\left({ }^{1}\right)$. Il le recuit longuement dans l'oxygène à I ooo puis à I $200^{\circ}$. $\mathscr{J}_{m}$ baisse et finit par atteindre la valeur $\mathfrak{y}_{m}=\mathrm{I}(\sigma=0, \mathrm{I} 9)$ " nombre qui n'est pas éloigné, dit-il, de ce que l'on mesure pour une substance paramagnétique ordinaire dans les mêmes conditions ". Ce résultat a été interprété par L. Néel comme une preuve que les ions ferreux causent le ferromagnétisme de $\mathrm{Fe}_{2} \mathrm{O}_{3} \alpha$ et que leur oxydation amène sa disparition.

Mais les phénomènes ne sont pas si simples. Le résultat de Hüttig et Kittel, contraire à celui de Snoëk, le laisse tout au moins supposer. Nous avons donc repris ces essais sur des oxydes d'histoire connue et nous avons tracé les courbes complètes d'aimantation au lieu de mesurer une seule constante [42].

${ }^{\circ}$ Un oxyde provenant de la calcination du sulfate ferrique à $800^{\circ} \mathrm{C}$ fournit la courbe d'aimantation 1 (fig. 8) avec $\sigma_{m}=0,33$ et un champ coercitif

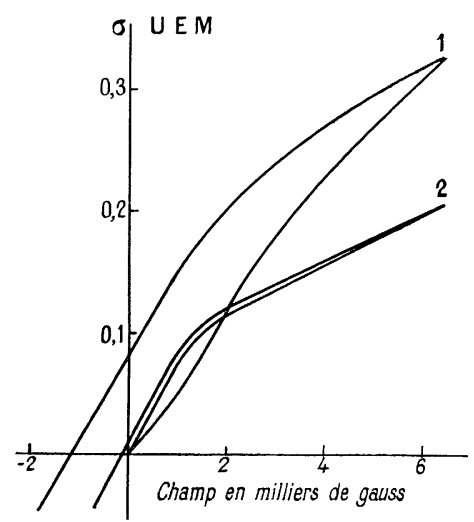

Fig. 8. - Oxyde ferrique dérivé du sulfate. Influence d'un recuit dans l'oxygène à i I $00^{\circ} \mathrm{C}$.

de 1000 gauss environ. On chauffe $\mathrm{I} h$ à $1000^{\circ}$ et $2 \mathrm{~h}$ à $\mathrm{I} \mathrm{IOO}^{\circ}$ dans l'oxygène à la pression atmosphérique. La valeur de $\sigma_{m}$ baisse peu à peu jusqu'à 0,20 et s'y maintient après un chauffage

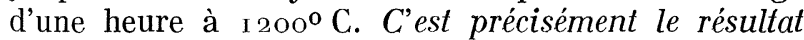
de Sno ëk. Mais la courbe d'aimantation tracée montre que le ferromagnétisme subsiste (fig. 8, courbe 2). L'hystérèse est considérablement réduite. Le champ coercitif est tombé à une centaine de gauss. Au delà de 2 ooo g, la courbe d'aimantation est pratiquement rectiligne et réversible de pente $\%=19,7 \cdot 10^{-i ;}$, conformément à ce que nous avons dit sur la susceptibilité réversible.

L'oxydation a réduit l'hystérèse, mais n'a pas fait disparaître le ferromagnétisme.

(1) Ce nombre est beaucoup plus élevé que les valeurs courantes qui ne dépassent pas 0,5 . Il est possible que cet oxyde contint de la magnétite libre. $2^{\circ}$ Dans un deuxième essai, nous avons chauffé, dans l'oxygène à $\mathrm{I}$ I $00^{\circ}$ pendant $2 \mathrm{~h}$, l'oxyde dérivé du nitrate qui a fourni la courbe 3 ( $f i g$. 4). L'évolu-

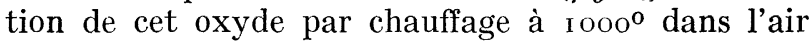
a fait passer la courbe d'aimantation progressivement de la forme 2 à la forme 3 où elle s'est fixée. Le chauffage à I $100^{\circ}$ la porte à la forme 4 comme si la présence d'oxygène était sans influence. L'évolution observée par chauffage dans l'air s'est poursuivie, l'hystérèse et l'aimantation maximum ont continué à croître, résultat tout à fait contraire au précédent. On a pensé que la structure granuleuse de l'oxyde n'était pas favorable à la 'pénétration de l'oxygène, on l'a donc pulvérisé.

$3^{0}$ Le broyage au mortier d'agate a été poussé aussi loin que possible. L'oxyde primitivement granuleux et noir est devenu rouge violacé. Ses propriétés magnétiques ont considérablement diminué : $\sigma_{m}$ est tombé à o, 5 U. E. M., $H_{\text {c }}$ est passé de 3 ooo à 1300 gauss ( $\mathrm{fig} .9$, courbe 5). Cet oxyde fut alors

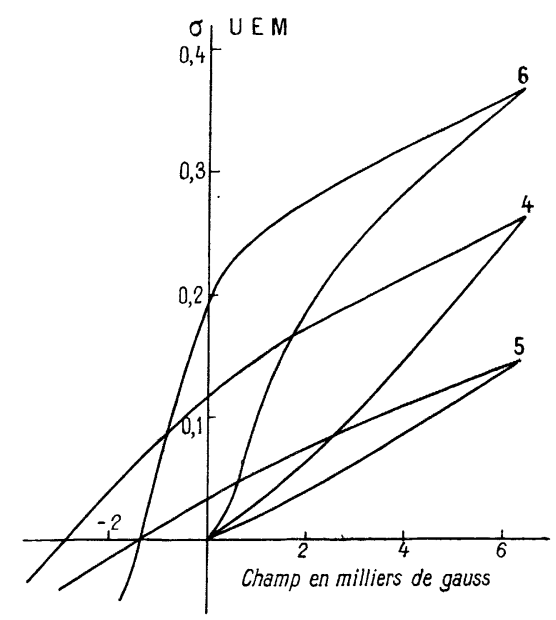

Fig. 9. - Oxyde ferrique dérivé du nitrate.

Suite de l'évolution de la figure 4. L'oxyde est broyé (courbe $\mathrm{n}^{\circ} 5$ ), puis- recuit dans l'oxygène à 1 I $^{\circ} \mathrm{C}$ (courbe $\left.n^{0} 6\right)$.

chauffé $2 \mathrm{~h}$ à I I oo dans l'oxygène. Accroissement énorme des propriétés magnétiques : la courbe d'aimantation passe de la forme 5 à la forme 6 . Le coefficient d'aimantation initial passe de 20 à $100.1^{-6}$. C'est le résultat de Hüttig et Kuttel. Les causes en sont probablement multiples : disparition des déformations permanentes causées par le broyage et accroissement de taille des cristaux; adsorption d'oxygène facilitée par la finesse de la poudre.

$4^{\circ}$ Enfin, nous avons soumis un oxyde dérivé du nitrate à basse température $\left(400^{\circ}\right)$ fournissant une courbe d'aimantation analogue à 1 ( $f i g$. 4), à un chauffage de 2 h à $1100^{\circ}$ dans l'oxygène. Nous avons 
retrouvé sensiblement la même courbe 3 que dans l'air.

Comme conclusion, nous voyons que le recuit dans l'oxygène peut amener aussi bien une croissance qu'une décroissance des propriétés magnétiques. Dans aucun cas, il ne fait disparaître le ferromagnétisme. Il semble que l'excès comme le déficit d'oxygène dans le réseau de $\mathrm{Fe}_{2} \mathrm{O}_{3} \alpha$ exalte ce ferromagnétisme. Mais cette question n'est pas résolue. Elle exige des expériences soignées qui restent à faire.

9. Influence du broyage. - La diminution des propriétés magnétiques par simple broyage, passage de la courbe 4 à la courbe 5 (fig. 9), est un phénomène important que nous allons étudier sur des poudres à grains monocristallins.

Nous l'avons observé sur tous les oxydes chauffés plusieurs heures à $1000^{\circ}$ et nous l'attribuons à la fragmentation des cristaux.

$\mathrm{Au}$ contraire, la pulvérisation d'un oxyde préparé à basse température (calcination du nitrate à $400^{\circ}$ ) n'amène aucune variation des propriétés magnétiques, parce que les monocristaux sont trop petits pour être atteints par la pulvérisation.

On pourrait aussi penser attribuer cette diminution à l'oxydation du fer ferreux, mais alors on ne comprendrait pas qu'un chauffage de $2 \mathrm{~h}$ dans l'oxygène à I I $0^{\circ}$ ne produise pas d'oxydation (passage de la courbe 3 à la courbe $4, f i g .4$ ) et qu'un broyage poussé dans l'air à la température ordinaire en produise une importante (passage de la courbe 4 à la courbe 5 , fig. 9). Nous ne croyons pas que dans le cas actuel, cette dernière explication doive être retenue.

\section{II. - Monocristal d'hématite. Poudres de monocristaux.}

On ne possède que des monocristaux naturels de $\mathrm{Fe}_{2} \mathrm{O}_{3} \alpha$ qui soient assez gros pour permettre une mesure magnétique sur un individu unique. Les valeurs absolues des aimantations dans un champ donné sont variables, l'existence et l'étendue de l'hystérèse changent d'un cristal à l'autre. Rien ne prouve que dans bien des cas la présence d'un peu de magnétite libre n'ait pas compliqué les phénomènes, car les auteurs n'ont pas déterminé les points de Curie de leurs échantillons [2, 4, 7]. Il semble que les nombres les plus faibles offrent le plus de garantie. Nous nous limiterons pour cette raison aux résultats de T. Townsend Smith [7].

10. Monocristal d'hématite. - On sait que ce cristal dérive d'un rhomboèdre primitif de faces culminantes $86^{\circ}$ environ. L'axe ternaire est de révolution pour les propriétés magnétiques. Voici đ'ailleurs comment' l'auteur résume ces propriétés :

I ${ }^{\circ}$ La symétrie de l'hématite comporte, au point de vue magnétique, un axe de symétrie avec un plan perpendiculaire dans lequel la susceptibilité est indépendante de l'orientation.

$2^{\circ}$ Dans ce plan principal l'hématite est ferromagnétique et présente de l'hystérèse. L'intensité d'aimantation est faible et n'approche pas de la saturation dans des champs de 4000 gauss.

3o Parallèlement à l'axe les spécimens de beaucoup de localités différentes sont paramagnétiques, mais quelques échantillons de l'île d'Elbe présentent une forte hystérèse comme Westmann [2] l'a trouvé également, en travaillant dans des champs faibles avec une hématite de Kragerö. Dans tous les cas, toutefois, et pour l'intervalle des champs utilisés, l'intensité d'aimantation suivant l'axe est beaucoup plus faible que celle dans le plan principal.

$4^{\circ}$ Pour les directions intermédiaires, l'aimantation n'est plus dirigée suivant le champ et pour certaines directions le couple exercé sur la matière est relativement grand. La composante parallèle au champ est maximum ou minimum pour des orientations à $90^{\circ}$ l'une de l'autre. La composante normale au champ n'a pas été mesurée.

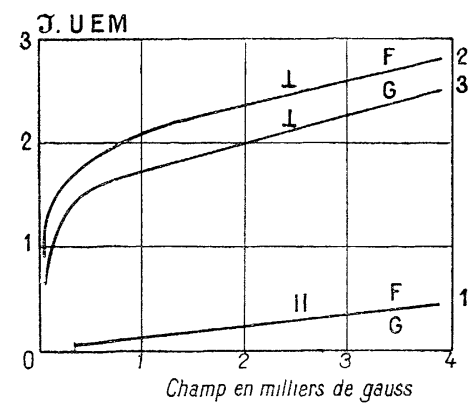

Fig. 10. - Aimantation d'un monocristal d'hématite suivant l'axe $(||)$ et normalement à l'axe $(\perp)$. D'après Townsend Smith [7].

Ce sont les valeurs de l'intensité d'aimantation qui sont portées en ordonnée. Pour avoir $\sigma$, diviser par 5,2.

Nous reproduisons (fig. Io) les courbes de première aimantation suivant les directions principales, dans le cas le plus simple du paramagnétisme suivant l'axe.

La droite 1 représente précisément cette loi linéaire d'aimantation suivant l'axe pour deux cristaux particuliers G et F. Elle correspond à un coefficient d'aimantation $\%=2 \mathrm{I} \cdot \mathrm{IO}^{-6}$ sensiblement identique au coefficient d'aimantation réversible des formes de $\mathrm{Fe}_{2} \mathrm{O}_{3} \alpha$ à grains polycristallins.

Les courbes 2 et 3 sont les lois d'aimantation pour ces deux cristaux dans le plan normal à l'axe. Elles sont à peu près rectilignes entre ı 000 
et 4 ooo gauss et de pente $\%=40.10^{-6}$. Comme il y a hystérésis, le coefficient sur la courbe de retour est plus faible, mais nous ignorons sa valeur. La droite 2 prolongée fournirait dans un champ de 65 oo gauss, $\sigma_{m}=0,65$.

La première ascendante des oxydes à grains polycristallins peut avoir une forme bien différente

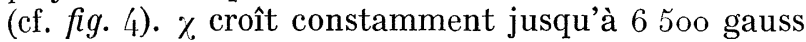
et $\sigma_{m}$ est beaucoup plus petit $\left(\sigma_{m} \not \neq 0,2\right.$ à 0,3$)$. Quelquefois pourtant le maximum de $\chi$ est atteint dès l'origine et l'on se rapproche de la forme 2 . A noter, par exemple, l'analogie pour la courbe 2 (fig. 8).

11. Fragmentation du réseau [36]. - Les propriétés magnétiques des poudres obtenues par fragmentation de monocristaux sont fort curieuses et permettent d'interpréter divers résultats. On est parti soit de cristaux naturels de grande taille, soit de petits cristaux synthétiques formés dans le borax fondu. Dans tous les cas, on a vérifié par enregistrement thermomagnétique que la substance de départ ne contenait pas de magnétite libre. Les monocristaux ont été pulvérisés, tamisés, lévigés dans l'alcool de façon à obtenir des fractions à grains moyennement identiques. La taille moyenne d'un grain d'une fraction donnée a été déterminée au microscope.

Il y a lieu d'insister sur le point suivant : jusqu'à io $\mu$ les poudres sont d'un beau noir; le grain est de toute évidence un fragment détaché du monocristal. Le microscope montre ses arêtes vives et il n'y a pas de raison pour que la structure réticulaire intérieure ne soit pas identique à celle du monocristal. Il y a bien eu fragmentation, non broyage et l'oxydation du fer ferreux intérieur, s'il en existe, ne saurait être invoquée. La variation des propriétés magnétiques jusqu'à ro $\mu$ ne peut donc être attribuée qu'à la diminution de taille des monocristaux. Au-dessous de ro $\mu$, la poudre devient violacée, puis d'un beau rouge vif pour I à $2 \mu$. Les grains ne se maintiennent plus distincts au séchage, mais tendent à former des amas confus. Il faut un broyage poussé pour aboutir à ces dimensions et rien ne prouve, a priori, que le réseau n'a pas été déformé, qu'une couche isotrope n'a pas recouvert les grains, que les ions ferreux présumés n'ont pas été partiellement oxydés. Un diagramme $\mathrm{X}$ des poudres les plus fines donne les raies de $\mathrm{Fe}_{2} \mathrm{O}_{3} \alpha$ très nettes, mais naturellement très élargies. La couche isotrope ne peut donc occuper qu'une petite fraction du grain. Il est possible que de nouvelles causes interviennent pour produire les variations magnétiques dans ces dimensions, mais il n'y a pas de raison pour que la cause principale ne reste pas la fragmentation du réseau.

Ayant donc réalisé des poudres de monocristaux de $\mathrm{Fe}_{2} \mathrm{O}_{3} \alpha$ telles que les grains d'une poudre donnée soient tous de même dimension moyenne $d$, on a construit la courbe d'aimantation de chaque échantillon et l'on a pu suivre l'influence de $d$ sur les propriétés magnétiques de ces poudres.

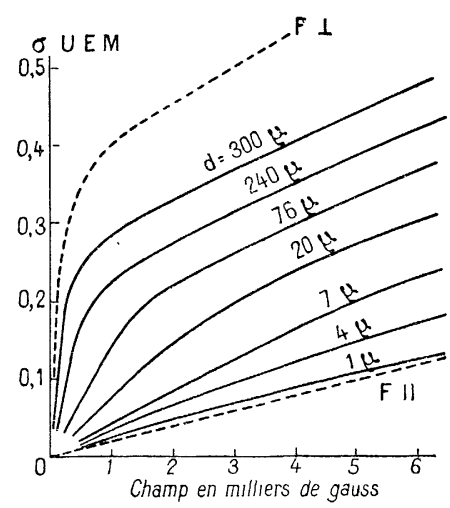

Fig. I1. - Poudres de monocristaux de $\mathrm{Fe}_{2} \mathrm{O}_{3} \alpha$. Influence du diamètre $d$ des grains. Champs croissants.

Les courbes en pointillé représentent l'aimantation du cristal d'hématite $\mathrm{F}$ suivant l'axe $(\mid)$ et normalement à l'axe ( 1 ).

Les courbes de première aimantation forment le réseau de la figure i s sur laquelle on a tracé, en pointillé, les courbes 1 et 2 de Townsend Smith. L'influence de $d$ est considérable. Quand le grain est suffisamment gros, la première ascendante est proche de la courbe 2 de T. Smith (ferromagnétisme dans le plan normal à l'axe); elle s'abaisse quand $d$ diminue, devient de plus en plus rectiligne et finit par se confondre avec la droite 1 de T. Smith (paramagnétisme suivant l'axe). Cette insertion du réseau entre les lois d'aimantation suivant les directions principales est très frappante et doit exprimer un caractère essentiel du ferromagnétisme de $\mathrm{Fe}_{2} \mathrm{O}_{3} \alpha$.

Voici quelques chiffres : la susceptibilité spécifique initiale $\chi_{i}$ décroît rapidement avec $d$. Le coefficient I $0^{6} \chi_{i}$ qui est de l'ordre de I 000 à I 500 pour des grains de $300 \mu$ taillés dans des lamelles d'hématites synthétiques, tombe à 25 ou 30 pour une poudre de grains de l'ordre de $\mathbf{s} \mu$.

On n'observe aucune saturation dans un champ de 6500 gauss. La courbe d'aimantation dans les champs forts monte régulièrement avec une pente presque constante de l'ordre de $\chi_{l}^{\prime}=25$. Io $^{-6}$. A 6 5̃oo gauss, l'aimantation maximum atteinte pour des grains de 3 oo $\mu$ ne dépasse pas o,5 U.E.M. Elle s'abaisse à o, 3 pour des grains de $\mathrm{I} \mu$.

Le réseau des courbes d'aimantation par champs décroissants est tracé sur la figure 12 . Le trajet de retour, au début, se détache fort peu de la courbe d'aller. Sa pente, indépendante de la taille des grains et de l'origine de la poudre, a en moyenne la valeur $\chi_{l}^{\prime \prime}=20.10^{-6}$. Ce résultat est remarquable, nous retrouvons la susceptibilité spécifique réversible de $\mathrm{Fe}_{2} \mathrm{O}_{3} \alpha$ étudiée au paragraphe 6 . 
Comme le trajet dans les champs forts est presque réversible pour ces poudres à grains monocristallins, $\%_{l}^{\prime}$ et $\%_{l}^{\prime \prime}$ sont très proches, mais c'est $\%_{l}^{\prime \prime}$ qui doit se rapprocher le plus du coefficient d'aimantation réversible.

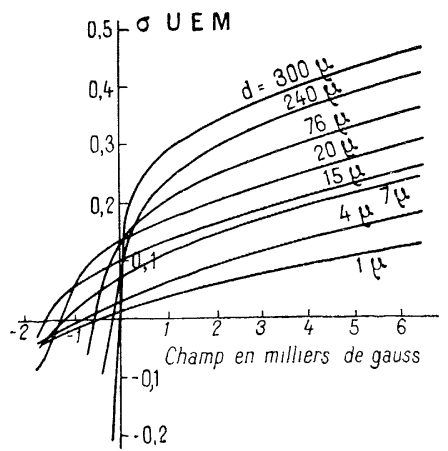

Fig. I 2. - Poudres de monocristaux de $\mathrm{Fe}_{2} \mathrm{O}_{3} \alpha$. Influence du diamètre $d$ des grains Champs décroissants. Hystérèse.

Dans les champs faibles, la courbe de retour est très différente de la courbe d'aller. La poudre présente, en général, une forte hystérèse qui dépend également de la taille de ses grains. L'aimantation rémanente $\sigma_{r}$, et le champ coercitif $H_{c}$ croissent d'abord quand la taille des grains diminue, passent par un maximum et deviennent ensuite de plus en plus faibles quand on s'approche des grains les plus fins. Le champ coercitif de l'ordre de roo à 200 gauss pour des grains de $300 \mu$ s'élève progressivement à mesure que $d$ diminue et atteint son maximum, 1800 gauss, pour des grains de 5 à ı o $\mu$. La décroissance est ensuite abrupte et les valeurs trouvées sont irrégulières. Pour des particules de l'ordre de I à $2 \mu$, nous avons observé des nombres variant de 400 à I 500 gauss. Il est possible que pour ces grains très fins la fragmentation du réseau n'intervienne pas seule, comme nous l'avóns exposé plus haut.

De tout cela il résulte que le caractère ferromagnétique des poudres de monocristaux de $\mathrm{Fe}_{2} \mathrm{O}_{3} \alpha$ s'atténue à mesure que les grains deviennent de plus en plus fins et qu'à la limite on aboutirait à un oxyde pamaragnétique comme celui étudié dans le paragraphe 2. Ceci s'explique si le ferromagnétisme dans le plan normal à l'axe est d'autant plus faible que les dimensions moyennès du cristal dans ce plan sont plus petites et si l'on admet qu'il disparaît pour des dimensions suffisamment réduites. Malheureusement, on ne voit pas comment cette hypothèse pourrait s'insérer dans notre représentation actuelle du ferromagnétisme.

12. Comparaison des deux types de poudres. - Peut-on ramener les propriétés magnétiques des poudres d'origine chimique à celles des poudres de grains monocristallins?

Il existe d'abord deux constantes communes à toutes les structures de $\mathrm{Fe}_{2} \mathrm{O}_{3} \alpha$, valables pour les monocristaux macroscopiques et pour tous les types de poudres, c'est la coefficient d'aimantation réversible dans les champs élevés, qui est constant et voisin de $\chi_{r}=20.10^{-6}$ et le point de Curie ferromagnétique $0=675^{\circ} \mathrm{C}$. Les autres propriétés qui mettent en jeu l'aimantation irréversible sont profondément différentes. La première ascendante de la courbe 2 ( fig. 4) n'a aucun rapport avec l'une quelconque des courbes du réseau de la figure i I. Le champ coercitif de 3 ooo gauss de la courbe 3 (fig. 3) dépasse largement les $\mathbf{} 800$ gauss qui marquent le champ coercitif maximum des poudres de monocristaux. D'une façon générale, la taille des éléments monocristallins n'explique pas les propriétés des poudres d'origine chimique. D'autres facteurs tels que l'imperfection de l'édifice réticulaire, les tensions internes, les gaz adsorbés, spécialement $\mathrm{O}_{2}$ peuvent intervenir. Mais nous ne savons rien de certain à ce sujet.

\section{III. - Essais d'interprétation.}

Nous voudrions dans cette dernière partie insister sur les propriétés dont on possède une explication chez les autres ferromagnétiques et chercher jusqu'à quel point de telles propriétés peuvent être considérées comme interprétées chez l'oxyde ferrique rhomboédrique.

13. Influence des dimensions des grains. a. Aimantation spontanée. - La loi d'aimantation d'une poudre de monocristaux dans des champs assez forts pour qu'il $y$ ait réversibilité s'écrit

$$
\sigma=\sigma_{0}+\chi H,
$$

où $\%=20.10^{-6}$ est une constante spécifique de $\mathrm{Fe}_{2} \mathrm{O}_{3} \alpha$ et indépendante en particulier des dimensions des grains, tandis que $\sigma_{0}$ l'aimantation spontanée en dépend énormément. Cette grandeur de l'ordre de o,4 pour des grains de quelques centaines de microns s'annule pour des grains de l'ordre de i $\mu$. La valeur critique à partir de laquelle elle commence à varier rapidement est comprise entre 5 o et ioo $\mu$ [29]. Ce phénomène est un aspect du ferromagnétisme de $\mathrm{Fe}_{2} \mathrm{O}_{3} \alpha$. Cela résulte du fait que cette variation est régulière et que le réseau des courbes d'aimantation admet comme limite supérieure la loi d'aimantation de l'hématite normalement à l'axe, comme limite inférieure la loi d'aimantation suivant l'axe; une semblable coïncidence ne saurait être fortuite et attribuée à l'oxydation du fer ferreux présumé (Cf. § 11). Aucune explication satisfaisante de ce phénomène n'a encore été proposée. 
b. Champ coercitif. - Cette grandeur dépend aussi des dimensions des grains. Là nous avons un phénomène analogue que L. Néel et ses élèves ont étudié sur des poudres métalliques [39]. A partir d'une dimension critique, le champ coercitif croît quand la taille des grains diminue. Une poudre de fer en grains de quelques' centièmes de microns atteint un champ coercitif de rooo gauss [40].

D'autre part, Ch. Guillaud [35] pour des alliages Mn-Bi a trouvé des champs coercitifs variant approximativement en raison inverse du diamètre des grains et atteignant la valeur énorme de I 2 ooo gauss pour un diamètre de $3,3 \mu$.

L'explication est la suivante : le processus habituel de désaimantation par déplacement des parois qui séparent les domaines élémentaires ne peut plus jouer quand les grains ont un diamètre de même ordre que l'épaisseur des parois. Les spins sont alors tous parallèles dans l'étendue d'un grain et la désaimantation exige leur retournement, ce qui nécessite un champ beaucoup plus fort que le champ coercitif habituel.

Un phénomène analogue se présente pour les poudres de monocristaux d'hématite quand les grains atteignent la dimension critique de 50 à $100 \mu$; le champ coercitif qui vaut alors quelques centaines de gauss, commence à croître et atteint I 700 gauss pour des grains d'une dizaine de microns. $\mathrm{Au}$ delà nous avons observé, dans certains cas, une décroissance de $H_{\text {, }}$, mais le phénomène est irrégulier et demande confirmation.

Dans les poudres à grains polycristallins d'origine chimique d'autres facteurs que la taille des monocristaux élémentaires interviennent pour déterminer le champ coercitif comme le montrent les faits suivants :

$\mathrm{I}^{0}$ L'existence d'un oxyde $\mathrm{Fe}_{2} \mathrm{O}_{3} \alpha$ rigoureusement paramagnétique, donc sans champ coercitif, quoique en grains très inférieurs à I $\mu(\S 2)$;

$2^{0}$ L'augmentation du champ coercitif par recuit ( $\S 3$ ), quoique les grains ne puissent que grossir par ce processus;

$3^{\circ} \mathrm{La}$ diminution du champ coercitif par broyage ( $\$ 9)$, malgré la diminution nécessaire de la taille des grains;

$4^{\circ} \mathrm{La}$ valeur absolue du champ coercitif, qui a atteint dans nos expériences jusqu'à 3 ooo gauss.

Mlle J. Roquet [38] a trouvé, d'autre part, qu'un champ de 7600 gauss était nécessaire pour faire disparaître l'aimantation permanente d'un échantillon d'oxyde soumis à un champ inducteur de 32000 gauss. Mais cette valeur n'est pas le champ coercitif qui est nécessairement plus faible. Elle s'accorde très bien avec un champ coercitif de 3 à 4 ooo gauss.

L'interprétation de ces faits est encore prématurée.
14. Aimantation à chaud. Hystérèse de température. Thermorémanence [31,33]. - Si l'on chauffe un échantillon de $\mathrm{Fe}_{2} \mathrm{O}_{3} \alpha$ dans un champ fixe d'une centaine de gauss, la courbe thermomagnétique $\sigma(T)$ obtenue présente l'une des formes de la figure 13 [31]. En général, $\sigma$ reste à peu près constant jusqu'à $500^{\circ} \mathrm{C}$, puis se met à croître d'une façon plus ou moins abrupte, passe par un maximum vers $63 \mathrm{o}^{\circ}$, puis décroît brusquement à $675^{\circ}$. Cette température est un véritable point de Curie. La substance perd alors son ferromagnétisme et présente, pour les températures supérieures, un paramagnétisme constant [22] $\chi=19,7 \cdot 10^{-6}$.

Chez certains échantillons, le maximum n'apparaît pas (fig. i $3 c$ ). La courbe de retour se détache

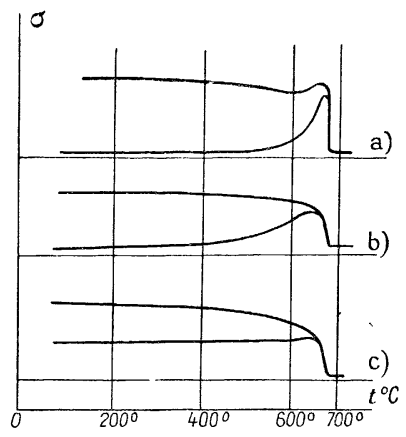

Fig. 13. - Courbes thermomagnétiques de $\mathrm{Fe}_{2} \mathrm{O}_{3} \alpha$. Formes diverses.

de la courbe d'aller peu au-dessous du point de Curie; un moment magnétique permanent est acquis et l'aimantation reste à peu près constante jusqu'à la température ordinaire. Dans quelques cas, $\sigma$ subit une légère diminution après le maximum (fig. ı $3 a$ ). L'existence d'un moment permanent acquis tout le long de la courbe d'aller et non pas seulement après passage par le point de Curie est bien mis en évidence par les expériences d'Esmaïl-Béguy (fig. I4) [31].

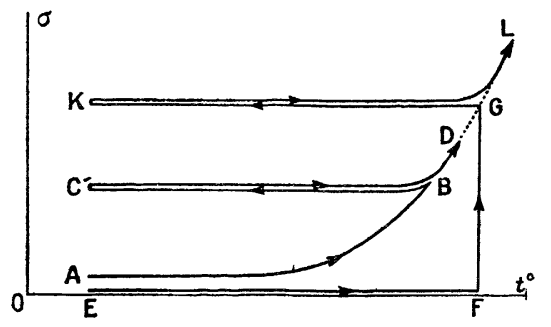

Fig. I4. - Thermorémanence de $\mathrm{Fe}_{2} \mathrm{O}_{3} \alpha$.

Ayant effectué le trajet $\mathrm{AB}$ arrêtons-nous au point $B$ et faisons décroître la température en maintenant le champ constant, nous décrivons la droite $\mathrm{BC}$ réversible. $\mathrm{Au}$ retour en $\mathrm{B}, \sigma$ reprend sa marche 
ascendante et la nouvelle courbe $\mathrm{BD}$ poursuit $\mathrm{AB}$.

Le moment permanent est indépendant de l'application progressive du champ. Si, par exemple, on chauffe en champ nul jusqu'en $F$ et qu'à cette température on applique brusquement le champ, $\sigma$ monte instantanément en $\mathrm{G}$ et la courbe de refroidissement suivant GK montre que le moment acquis est bien permanent.

${ }_{2}{ }^{\circ}$ L'allure des variations de $\sigma$ avec $T$ sur la courbe d'aller rappelle tout à fait les résultats obtenus pour les aciers. Les courbes de Hopkinson sont classiques [1]. Nous reproduisons ( fig. i 5) le

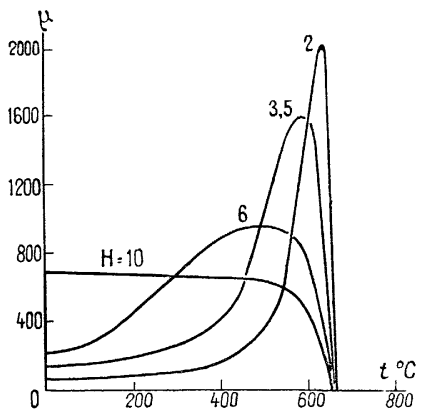

Fig. ı 5. - Courbes thermomagnétiques d'un acier au tungstène D'après Wills [3].

graphique de R. L. Wills [3] fournissant la perméabilité $\mu$ à champ constant en fonction de la température pour un acier au tungstène. Ces courbes représentent aussi $\mathcal{B}$ à un facteur constant $\frac{\mathrm{I}}{I}$ près, donc $\mathcal{J}$ qui lui est pratiquement identique et nous pouvons les considérer comme fournissant $\sigma(T)$ en valeur relative. Dans les champs faibles de l'ordre de 2 gauss, $\sigma$ passe par un maximum très accusé au voisinage du point de Curie. A mesure que le champ augmente, ce maximum baisse et s'étale. Il disparaît pour une dizaine de gauss. L'analogie des courbes thermomagnétiques de $\mathrm{Fe}_{2} \mathrm{O}_{3} \alpha$ (fig. I 3 ) et du réseau de Wills est très remarquable et l'on peut croire qu'une augmentation suffisante du champ appliqué ferait passer la courbe d'aller de la forme $a$ à la forme $c$. La théorie suivante due à L. Néel [41] permet d'en comprendre la cause.

$3^{\circ}$ La loi d'aimantation du fer dans les champs faibles, étudiée expérimentalement par Lord Rayleigh en 1887 , puis fondée théoriquement et précisée par L. Néel [37] s’écrit

$$
\sigma=\sigma_{\varsigma}\left(k_{1} \frac{h}{\boldsymbol{H}_{c}}-k_{2} \frac{h^{2}}{\boldsymbol{H}_{c}^{2}}\right)
$$

où $k_{1}$ et $k_{2}$ sont deux constantes numériques variables avec les échantillons et de l'ordre de quelques dixièmes; $\sigma_{\text {s }}$ et $H_{c}$ sont respectivement la saturation absolue et le champ coercitif à la température $T$.
Cette relation n'est valable que si le champ appliqué $h$ est petit devant $H_{c}$. Elle représente le lieu des sommets des cycles symétriques.

Voyons tout d'abord comment cette loi d'aimantation permet de concevoir une courbe thermomagnétique d'aller croissant avec la température [41]. Nous partons de la température ordinaire. Le champ appliqué $h$ restera constant pendant toute l'expérience. Le champ $H_{\text {c }}$ est par hypothèse grand par rapport à $h$. Faisons croître $T$; $\sigma_{s}$ et $H_{c}$ diminuent, ces deux grandeurs s'annulant au point de Curie. Mais $H_{c}$ diminue beaucoup plus vite que $\sigma_{\text {s }}$ en sorte que $\frac{\sigma_{s}}{\boldsymbol{H}_{c}}$ et surtout $\frac{\sigma_{s}}{\boldsymbol{H}_{c}^{2}}$ sont des fonctions croissantes de $T$. Dans un champ appliqué $h$ petit devant $H_{c}, \sigma$ doit croître avec $T$. Telle est l'origine de la partie montante de la courbe thermomagnétique.

Puis quand $H_{c}$ en diminuant est devenu beaucoup plus petit que $h$, la relation de Néel cesse d'être exacte, mais $\sigma$ est alors de l'ordre de $\sigma_{s}$ et diminue comme cette grandeur. Il existe donc une température pour laquelle $\sigma$ est maximum, c'est à peu près celle pour laquelle le champ coercitif devient égal au champ appliqué $h$.

Supposons maintenant que $H_{c}$ soit comparable à la température ordinaire au champ appliqué $h$, $\sigma$ ne présentera plus de maximum, mais restera constant avec $T$.

Dans tous les cas, à $67 \tilde{\partial}^{\circ} \mathrm{C}, \sigma$ décroîtra brusquement; la substance perdra son ferromagnétisme et deviendra paramagnétique. On sait que cette température est un véritable point de Curie.

La transformation des courbes de Wills avec le champ appliqué (fig. i 5) est conforme à cette explication en admettant un champ coercitif de l'ordre de ro gauss pour l'acier au tungstène utilisé. Les trois types de courbes d'aller de la figure I3 se comprennent également dans cette théorie, mais aucune expérience systématique sur l'influence du champ n’a encore été faite. Que peut-on prévoir pour les courbes de retour? Dans la substance vierge, les domaines élémentaires de Weiss sont aimantés dans toutes les directions et donnent un moment nul dans un champ nul. Dans le champ $h$, les déplacements de parois, puis les rotations, créent, suivant le champ, un moment appréciable qui croît avec la température pour $H_{c}$ grand devant $h$. Comme un domaine retourné dans le sens du champ conserve son orientation par refroidissement dans le même champ, le moment acquis à une certaine température reste permanent quand on revient à la température ordinaire. C'est bien ce que montrent les expériences d'Esmaïl (fig. I 4). Ceci se produit en particulier si l'on atteint le point de Curie, mais ce n'est pas nécessaire.

Signalons quelques faits que L. Néel interprète comme confirmant cette explication : 
a. If. Forestier [25] a trouvé que la courbe thermomagnétique d'aller ne présente un maximum que si les grains sont plus petits que $0,07 \mathrm{~mm}$, ce serait la taille pour laquelle le champ coercitif serait de l'ordre du champ appliqué. Comme $H_{\text {r }}$ croît quand les grains deviennent plus petits, le maximum s'accuserait avec la finesse des grains.

b. A. Michel [27] et [33] a trouvé, qu'après un recuit de $6 \mathrm{~h}$ à $\mathrm{I} 300^{\circ}$ le maximum disparaît. Mais à $1300^{\circ}$ la tension de dissociation devient suffisante pour faire apparaître des quantités notables de magnétite et l'interprétation semble bien délicate.

c. H. Forestier [25] a montré qu'un cristal d'hématite présente une thermorémanence prononcée quand le champ est dirigé suivant l'axe ternaire, et n'en présente pas, au contraire, quand le champ est dirigé normalement à l'axe, ce qui semble très paradoxal, puisque le cristal est paramagnétique suivant l'axe. L. Néel en donne une explication, mais le fait lui-même demande confirmation, car J. Kœnigsberger [21] trouve un résultat contraire. Il a montré, en I 932 , que dans un champ très faible de 0,4 gauss dirigé perpendiculairement à l'axe principal la thermorémanence est considérable et atteint la moitié de l'aimantation à saturation.

On se demande si le champ extérieur est bien le même dans les deux expériences de Forestier. Il est en effet nécessaire d'appliquer suivant l'axe un champ beaucoup plus fort que normalement à l'axe pour avoir une déviation comparable dans les deux cas; ne serait-ce pas la cause des différences de thermorémanence? La question reste pendante.

$4^{\circ}$ Disons enfin quelques mots du domaine paramagnétique au-dessus de $675^{\circ} \mathrm{C}$. En 1932, Mlle Serres [22] a trouvé sur deux échantillons entre 700 et $750^{\circ}$ un coefficient d'aimantation indépendant de la température $\chi=19,7 \cdot \mathrm{ro}^{-6}$. Ayant soupçonné que ce nombre pouvait dépendre de la préparation de l'oxyde, nous avons repris les mesures sur une vingtaine d'échantillons d'origines très différentes [30]. Nous avons retrouvé invariablement la même valeur.

En r937, K. Endo [32] a étudié la susceptibilité dans un domaine plus étendu $\left(700-\mathrm{I}^{\circ} \mathrm{oo}^{\circ} \mathrm{C}\right)$. Il trouve une légère influence de la température exprimable par une loi de Weiss : $\chi(T+3727)=0,085$, qui donne, par exemple, $\chi=18.10^{-6}$ à $725^{\circ} \mathrm{C}$. Si cette loi thermique était vraie dans tout l'intervalle des températures elle fournirait à $20^{\circ} \mathrm{C}$ exactement $\%=20.10^{-6}$ valeur identique à celle de Mlle Serres et à la susceptibilité réversible que nous avons attribuée à $\mathrm{Fe}_{2} \mathrm{O}_{3} \alpha$ à la température ordinaire. Cette coïncidence est évidemment favorable à l'hypothèse que le ferromagnétisme de l'oxyde rhomboédrique est superposé à un paramagnétisme de base régi par une loi unique à toute température.
15. Point de transition. - - A la température de $675^{\circ} \mathrm{C}$ la disparition du ferromagnétisme s'accompagne de deux anomalies importantes dont nous allons dire quelques mots.

G. Chaudron et H. Forestier [12, 17] en étudiant au dilatomètre de Chevenard, l'allongement en fonction de la température de bâtonnets d'oxyde ferrique aggloméré par pression, ont trouvé, vers $675^{\circ}$, une anomalie de dilatation rigoureusement réversible, qui révèle une discontinuité des coefficients de dilatation. Le phénomène s'étale sur un certain intervalle de température; il est tout à fait semblable à celui que présente la magnétite et son amplitude est sensiblement la même dans les deux cas. Si l'on se rappelle la différence considérable entre les propriétés magnétiques des deux substances, l'égale importance de l'anomalie dilatométrique est paradoxale comme les auteurs le font remarquer.

A cette même température $[14,19] \mathrm{Fe}_{2} \mathrm{O}_{3} \alpha$ présente une anomalie de chaleur spécifique, son amplitude est encore comparable à l'anomalie habituelle des ferromagnétiques courants.

En résumé, l'oxyde ferrique rhomboédrique à $675^{\circ}$ présente les trois phénomènes suivants :

a. Disparition du ferromagnétisme;

b. Discontinuité des coefficients de dilatation;

c. Discontinuité de la chaleur spécifique.

Ce sont les caractères d'un point de transition et les trois discontinuités sont liées à une transformation de second ordre de $\mathrm{Fe}_{2} \mathrm{O}_{3} \alpha$, si toutefois nous leur supposons une même origine. La difficulté à laquelle se heurte cette hypothèse est la disproportion entre les phénomènes magnétiques qui restent très faibles par rapport aux ferromagnétiques courants (aimantation spontanée de o,5 U.E.M. au lieu de roo pour $\mathrm{Fe}_{3} \mathrm{O}_{4}$ ) et les anomalies de dilatation et de chaleur spécifique qui sont de même ordre que pour le fer ou le nickel. L. Néel suppose deux origines distinctes à ces discontinuités et il imagine un mécanisme expliquant pourquoi elles se produisent à la même température. Nous allons exposer l'essentiel de sa théorie.

16. Théorie de Néel. - L'existence dans les champs élevés d'une susceptibilité réversible constante et l'aspect du réseau des courbes d'aimantation de la figure I I relatif à une poudre de fragments cristallins de taille variable, suggèrent que le ferromagnétisme est dû à une impureté et que la masse de $\mathrm{Fe}_{2} \mathrm{O}_{3} \alpha$ apporte seulement le paramagnéıisme sous-jacent de coefficient d'aimantation $\chi=20 . \mathrm{Io}^{-6}$. L'aimantation dans les champs forts qui est de la forme $\sigma=\sigma_{0}+\chi H$ comporterait une aimantation spontanée $\sigma_{0}$ variable avec l'échantillon, due à l'impureté et une aimantation paramagnétique $\chi H$ qui serait la contribution propre de $\mathrm{Fe}_{2} \mathrm{O}_{3} \alpha$. 
Telle est l'hypothèse précisée par L. Néel pour rendre compte de l'ensemble des propriétés de l'oxyde rhomboédrique. Pour lui, $\mathrm{Fe}_{2} \mathrm{O}_{3} \alpha$ pur est antiferromagnétique et responsable dans l'oxyde courant des propriétés suivantes :

I ${ }^{\circ} \mathrm{A}$ haute température, du paramagnétisme variable mis en évidence par $\mathrm{K}$. Endo et régi par la loi de Weiss : $\chi(T+3725)=0,085$;

$2^{0} \mathrm{~A}$ basse température, du paramagnétisme sous-jacent : $\%=20 \cdot 10^{-6}$;

$3^{\circ}$ Des anomalies de dilatation et de chaleur spécifique, se traduisant par une discontinuité de ces grandeurs au point de transition $t=675^{\circ} \mathrm{C}$.

L'oxyde courant contient, outre $\mathrm{Fe}_{2} \mathrm{O}_{3} \alpha$ une impureté que L. Néel suppose être la magnétite. Mais l'anisotropie considérable du ferromagnétisme des monocristaux d'hématite et le point de Curie $\theta=675^{\circ} \mathrm{C}$ ne permettent pas de supposer que cette magnétite est sous forme cubique. L. Néel fait l'hypothèse hardie qu'elle est sous forme rhomboédrique et que ses cristaux sont orientés par rapport au réseau de $\mathrm{Fe}_{2} \mathrm{O}_{3} \alpha$.

La magnétite rhomboédrique massive est inconnue, mais il est vraisemblable d'admettre son existence comme phase de transition. On sait en effet depuis longtemps que si des cristaux de magnétite croissent sur de l'oligiste, la face d'accolement est toujours une face $a^{1}$ des deux espèces (normale à l'axe ternaire) et l'orientation des deux cristaux autour de l'axe commun est telle que les éléments de symétrie ternaire coïncident. Les atomes d'oxygène de la magnétite sont précisément disposés dans des plans $a^{1}$ aux nœuds d'un pavage hexagonal compact. La distance de deux proches voisins est $l^{\prime}=2,97 \AA$. La disposition des atomes d'oxygène de l'hématite est identique avec une distance $l^{\prime \prime}=2,90 \AA$ pour deux proches voisins. Il est naturel d'admettre que l'accolement se fait par un plan commun d'atomes d'oxygène avec une distance $l$, intermédiaire entre $l^{\prime}$ et $l^{\prime \prime}$.

Du côté hématite la dilatation dans le plan des $\mathrm{O}$, àccompagnée d'une contraction dans la direction perpendiculaire, ne change pas le caractère rhomboédrique du réseau, qui est seulement déformé et qui revient au réseau normal après quelques plans réticulaires. Du côté magnétite, la dilatation dans le plan des $\mathrm{O}$ s'accompagne d'une contraction dans la direction de l'axe ternaire qui transforme la maille cubique en maille rhomboédrique, avec de fortes tensions internes. La déformation s'atténue à mesure qu'on s'éloigne du plan d'accolement et à distance convenable on revient à la magnétite cubique.

Cette phase de transition que constitue la magnétite rhomboédrique doit prendre naissance identiquement dans les cristaux d'oxyde ferrique ordinaire qui contiennent du fer ferreux et où de la magnétite se sépare. Elle doit se développer sous forme de plaquettes minces de façon à réduire au minimum l'énergie de déformation élastique. Mais, étant donnée la dilution, on peut admettre que, dans ces plaquettes, le réseau cubique normal n'est jamais atteint, en sorte qu'un cristal d'oxyde ferrique courant serait une dispersion de plaquettes de magnétite rhomboédrique dans une matrice rhomboédrique de $\mathrm{Fe}_{2} \mathrm{O}_{3} \alpha$, avec coïncidence des axes ternaires et des éléments de symétrie normaux à ces axes. Cette structure hypothétique permet d'expliquer une partie des propriétés.

I ${ }^{\circ}$ La magnétite rhomboédrique sera ferromagnétique comme la magnétite cubique, puisque la coordination des atomes ne change pas et que les distances ferromagnétiques seront, à très peu près, les mêmes dans les deux cas. Si les aimantations spontanées sont également de même ordre, une concentration de $\mathrm{I} / \mathrm{Ioo}^{\mathrm{e}}$ environ expliquera l'aimantation spontanée de l'oxyde ferrique courant (o,5 U. E. M.).

$2_{2}^{\circ}$ Cette substance doit être anisotrope. L'axe qui est perpendiculaire au plan des plaquettes sera de difficile aimantation. La distribution isotrope des plaquettes dans le plan normal à l'axe, entraînera l'isotropie magnétique dans ce plan. Toute direction parallèle au plan des plaquettes sera de facile aimantation. Ce sont précisément les propriétés du cristal d'hématite dont les directions cristallographiques coïncident avec celles des inclusions de magnétite.

$3^{\circ}$ L'état d'extrême division de l'impureté expliquera l'hystérèse accentuée et le grand champ coercitif.

$4^{\circ} C^{\prime}$ 'est une coïncidence étonnante que le point de transition d'antiferromagnétisme de $\mathrm{Fe}_{2} \mathrm{O}_{3} \alpha$ et le point de Curie de la magnétite rhomboédrique soient à la même température $675^{\circ} \mathrm{C}$, alors qu'on s'attendrait à les trouver indépendants. L. Néel montre que la structure proposée explique cette coïncidence.

D'autres propriétés n'entrent pas dans ce cadre, en particulier l'évolution de la loi d'aimantation d'une poudre quand les grains deviennent de plus en plus petits (diminution régulière de l'aimantation spontanée, augmentation du champ coercitif suivie d'une diminution). Nous ne croyons pas que ces phénomènes soient secondaires comme le pense L. Néel. Nous avons montré qu'ils ne peuvent tenir ni à l'insuffisance du champ appliqué, ni à l'oxydation de l'impureté ferreuse. Nous pensons qu'ils représentent, au contraire, un aspect essentiel du ferromagnétisme de $\mathrm{Fe}_{2} \mathrm{O}_{3} \alpha$, qu'il faudra d'abord expliquer pour comprendre ce ferromagnétisme.

L'ingénieuse théorie de L. Néel postule d'ailleurs la présence de fer ferreux dans tout oxyde ferrique 
ferromagnétique et cette affirmation est certainement trop générale. Les expériences de Snoëk ne prouvent rien de semblable comme nous l'avons démontré au paragraphe 8 . Le recuit prolongé dans l'oxygène à I $\mathrm{I}_{0}{ }^{\circ}$ qui doit oxyder totalement le fer ferreux ne fait jamais disparaître le ferromagnétisme. Tantôt il l'atténue, tantôt il l'exalte. De plus, il existe des conditions de formation de $\mathrm{Fe}_{2} \mathrm{O}_{3}$ qui excluent la présence de fer ferreux. Un nitrate ferrique bien cristallisé, décomposé à basse température, ne peut pas contenir de fer ferreux; après recuit dans l'oxygène à la pression atmosphérique dans des conditions où la tension de dissociation est de l'ordre du centimètre de mercure, on ne conçoit pas davantage la formation de fer ferreux et pourtant le réseau des courbes de la figure 4 montre avec quelle intensité l'oxyde recuit présente les caractères du ferromagnétisme.

Le ferromagnétisme de $\mathrm{Fe}_{2} \mathrm{O}_{3} \alpha$ n'est donc pas lié à la présence de fer ferreux. Il nous semble nécessaire de le considérer comme une propriété spécifique de l'oxyde. Un déficit ou un excès d'oxygène par rapport à la composition stœchiométrique modifiera les caractères de ce ferromagnétisme, mais l'aimantation spontanée se produira même si la constitution est rigoureusement $\mathrm{Fe}_{2} \mathrm{O}_{3}$, à condition que les cristaux élémentaires ne soient pas trop petits : l'hypothèse est malheureusement assez vague, mais les faits à notre disposition ne permettent pas d'être plus catégorique.

Gonclusion. - Les propriétés magnétiques essentielles de $\mathrm{Fe}_{2} \mathrm{O}_{3} \alpha$ peuvent se résumer comme il suit : elles sont la superposition d'un paramagnétisme de base variant avec la température suivant la loi de K. Endo :

$$
\left.\begin{array}{rl}
\chi(T+3727) & =0,085 \\
\chi_{200 \mathrm{C}} & =20.10^{-6}
\end{array}\right\}
$$

et d'un ferromagnétisme d'intensité variable, qui disparaît à $\theta=675^{\circ} \mathrm{C}$. En ce même point 0 , se produisent simultanément des discontinuités du coefficient de dilatation et de la chaleur spécifique. Cette température est donc un point de transition d'une transformation de second ordre. Au-dessus de $\theta$ l'oxyde est paramagnétique pur; au-dessous paramagnétisme et ferromagnétisme sont superposés. Précisons leur caractère.

Dans les champs très élevés où l'aimantation est réversible, la superposition s'exprime par la loi simple :

$$
\sigma=\sigma_{0}+\gamma I I
$$

où $\sigma_{0}$ est l'aimantation spontanée ferromagnétique et $\chi H$ la contribution du paramagnétisme de Endo. L'aimantation spontanée dépend de la préparation et de l'histoire de l'oxyde, elle ne dépasse jamais ı U.E. M. On sait par exemple préparer des oxydes de ferromagnétisme inappré- ciable à la température ordinaire $\left(\sigma_{0}=0\right)$ et qui sont purement paramagnétiques de coefficient voisin de $20.10^{-6}$. Mais tous deviennent ferromagnétiques par recuit à haute température et leur paramagnétisme pur est exceptionnel.

Pour les oxydes courants soumis à des champs moyens, l'aimantation comporte une partie irréversible; les trajets d'aller et de retour ne sont plus superposés; l'hystérèse peut être considérable et le champ coercitif atteint parfois 3 ooo gauss. Pourtant, le paramagnétisme de Endo continue à se manifester par la valeur du coefficient d'aimantation réversible dans les champs supérieurs à quelques milliers de gauss. Ce coefficient est en effet constant et indépendant de l'origine et de l'histoire de l'échantillon. Sa valeur $\%=20.10^{-6}$ est identique à la susceptibilité paramagnétique, parce que la susceptibilité réversible ferromagnétique tend vers zéro dans les champs de cet ordre.

Les propriétés magnétiques des monocristaux de $\mathrm{Fe}_{2} \mathrm{O}_{3} \alpha$ apportent quelques données nouvelles. Ils sont rhomboédriques : paramagnétiques suivant l'axe ternaire, isotropes et ferromagnétiques dans le plan normal à cet axe. On retrouve comme coefficient du paramagnétisme suivant l'axe, la valeur habituelle $\chi=20.10^{-6}$ et normalement à l'axe la loi (2) avec une aimantation spontanée de l'ordre de 0,5 U. E. M.

Si l'on pulvérise l'un de ces cristaux et qu'on fractionne les grains de façon à grouper ensemble les éléments monocristallins de même taille (diamètre $d$ ) on constate que les propriétés d'une poudre dépendent de $d$. La loi d'aimantation dans les champs forts reste la loi (2) avec $\chi=20.10^{-6}$, mais l'aimantation spontanée de l'ordre de o,4 pour les gros grains, commence à diminuer rapidement autour de $d=$ roo $\mu$ pour devenir insensible autour de $d=\mathrm{I} \mu$. Il paraît difficile d'échapper à la conclusion que le ferromagnétisme ne se manifeste dans le plan normal à l'axe que si les dimensions du cristal dans ce plan sont suffisantes. Un cristal de i $\mu$ environ dans toutes les directions serait isotrope et présenterait le paramagnétisme de base à l'état pur. Ces propriétés très nettes des poudres de monocristaux n'ont pas encore permis d'interpréter les caractères si variables du ferromagnétisme des poudres de $\mathrm{Fe}_{2} \mathrm{O}_{3} \alpha$ d'origine chimique.

Une dernière question se pose : devons-nons attribuer le paramagnétisme de base à $\mathrm{Fe}_{2} \mathrm{O}_{3} \alpha$ et le ferromagnétisme superposé, qui reste toujours faible, à une impureté étrangère comme la magnétite rhomboédrique de L. Néel; ou bien devons-nous admettre que l'oxyde de composition stœchiométrique $\mathrm{Fe}_{2} \mathrm{O}_{3}$ présenterait déjà un ferromagnétisme spécifique? Il est difficile d'être catégorique sur ce point. Mais il nous semble que les faits présentés dans cette étude sont plus favorables à la seconde hypothèse. Le déficit ou l'excès d'oxygène dans le 
réseau de $\mathrm{Fe}_{2} \mathrm{O}_{3} \alpha$ peuvent modifier profondément son ferromagnétisme, mais ce ferromagnétisme même préexiste à la modification du réseau. Nous avons groupé dans cette conclusion les propriétés essentielles que devra interpréter toute théorie.

Remarque de $M$. Snoëk. - L'hystérèse dans les substances antiferromagnétiques est un phénomène presque général. Dans une note récente, j'ai indiqué comment les défauts de structure détruisent la cohérence, en produisant ainsi des domaines quasi indépendants, porteurs d'un moment permanent faible. Il faut probablement voir là l'origine des phénomènes observés.

Remarque de $M$. Forrer. - A l'appui de l'opinion de M. Snoëk, on peut remarquer que l'étude des distances ferromagnétiques et antiferromagnétiques dans les composés du fer et du manganèse montre que, dans le cas limite, une augmentation de distance de $\mathrm{o}, \mathrm{I} \AA$ suffit à transformer un anti en un ferromagnétisme. Dans certaines couches doubles perpendiculaires à l'axe de $\mathrm{Fe}_{2} \mathrm{O}_{3} \alpha$, la distance habituelle de 2,88 $\AA$ pourrait être augmentée de o, I $\AA$ par des impuretés et introduire ainsi un ferromagnétisme apparent, par défaut de compensation.

Remarque de M. Michel. - Je tiens, à propos du rapport de M. le Professeur Chevallier, à affirmer 'opinion qui a toujours été soutenue par MM. Chau- dron, Bénard et moi-même [44], à savoir que $\mathrm{Fe}_{2} \mathrm{O}_{3}$ ne possède que deux formes allotropiques et deux seulement : la forme $\alpha$ rhomboédrique et la forme $\gamma$ cubique.

En particulier :

${ }^{\circ}$ Le produit obtenu par recuit vers $650^{\circ}$ d'un mélange précıpité de $\mathrm{Fe}_{2} \mathrm{O}_{3}$ et $\mathrm{GlO}$ n'est qu'un mélange de forme $\alpha$ et de cubique stabilisé par GlO [33]. Si le diagramme $\mathrm{X}$ ne montre que la forme $\alpha$, c'est que la quantité de $\gamma$ est faible, quoique l'essai de dissociation sous vide la mette nettement en évidence.

$2^{\circ}$ La variété hexagonale (?), dite $\delta$ [34], n'est que du cubique très fin, mélangé à du rhomboédrique.

$3^{\circ}$ Le fait que la déshydratation de la goethite conduise à des échantillons de $\mathrm{Fe}_{2} \mathrm{O}_{3}$ doués d'une très faible aimantation pourrait s'interpréter en considérant que la déshydratation pulvérise très finement les grains d'oxyde : on sait, en effet, que des ferromagnétiques très fins perdent, tout au moins dans les champs faibles, la plus grande partie de leur aimantation à des températures très inférieures au point de Curie ordinaire.

Je tiens à rappeler également le point suivant [44] : la calcination du sel ferrique (nitrate, sulfate) fait apparaître de petites quantités de forme $\gamma$. La présence de cette forme est affirmée par le fait que, en présence d'un stabilisant, comme l'alumine [45], il se forme du sesquioxyde cubique stabilisé.

\section{BIBLIOGRAPHIE.}

[1] Hopkinson J. - Phil. Trans., i 889, 180 A, 443.

[2] Westmann J. - Diss. Upsala, 1897.

[3] Wills R. L. - Phil. Mag., I900, 50, I

[4] Kunz J. - N. Jahrb. f. Min., I 907, 1, 62.

[5] Berndt G. - Ann. Physik, i $908,27,712$.

[6] Honda K. et Soné T. - Sc. Rep. Tohoku. Imp. Univ., I $914,3,2.23$.

[7] Townsend Smith T. - Phys. Rev.. (2), i9i6, 8, 721 .

[8.9] Sosman R. B. et Hostetter J. C. - J. of Amer. Chem. Soc., г 9 1 $6,38,807$ et i 88.

10.11] Sosman R. B. et Hostetter J. C. - Trans. Amer. Inst. of mining eng., $1918, \mathbf{5 8}, 409$ et 434.

[12] Chaudron G. et Forestier H. - C. R. Acad. Sc., I $924,179,763$.

[13] Blanc L. et Chaudron G. - C. R. Acad. Sc., i 925 , 180, 289 .

[14] Furnas X. - Trans. Amer. Inst. Ch. Ing., 1926, 18, 309.

[15] Blanc L. - Thèse d' Université, Paris, I 926.

[16] Chevallier R. - C. R. Acad. Sc., 1927, 184, 674.

[17] Forestier H. - Thèse, Paris, i 928.

[18] Hugget J. - Thèse, Paris, I 928.

[19] Roth W. A. el Bertram W. - Z. Elektroch., I929, 35, 306 .

[20] Hüttig G. F. et Kittel H. - Z. an. allg. Ch., i 93 I, $199,129$.

[21] Kønigsberger J. - Physik, Z. 1932, 33, 468 et 763.

[22] Serres Mile A. - Ann. Physique, i 932 , (1 o), 17, 5.

[23] Forestier H. - C. R. Acad. Sc., 1 934, 199, 720.

[24] Michel A. et Chaudron G. - C. R. Acad. Sc., i 935, 200, 2171.
[25] Forestier H. - C. R. Acad. Sc., i $935,201,45$.

[26] Gottschalk V. - Physics, i 935,6, , 27.

[27] Mighel A. - C. R. Acad. Sc., i 936, 202, г 769 .

[28] SNö̈к J. L. - Physica, г 936, 3, 463.

[29] Chevallier R. et Mathieu Mie S. - C. R. Acad. Sc., I $937,204,854$.

[30] Chevallier R. et Esmail-Béguy Z.-Bull. Soc. Chim., (5), $1937,4,1735$.

[31] Esmail-BÉGuY Z. - Thèse d'Université, Nancy, i937. [32] Endo (K.). - Sc. Rep. Tohoku Imp. Univ., 1937, 25,879 .

[33] Mrchel A. - Ann. Chim., i 937, 8, p. 3 17; Thèse, Paris, 1937 .

[34] Glemser O. et Gwinner E. - Z an. allg. Chem, i 939 , 240, г61; Résumé dans Strukturbericht, 1 943, 7, 104.

[35] Guillaud Ch. - Thèse, Strasbourg, r 943.

[36] Chevaldier R. et Mathieu Mile S. - Ann. Physique, (1 I), $1943,18,258$.

[37] Néel L. - Cahiers de Physique, 1942, nº 12 et 1943, $\mathrm{n}^{\circ} 13, \mathrm{i} 8$.

[38] Roquet Mile J. - C. R. Acad. Sc., i947, 224. I418.

[39] Néel L. - G. R. Acad. Sc., r $947, \mathbf{2 2 4}, 1488$ et 1550.

[40] Weil L. - C. R. Acad. Sc., r947, 225, 229.

[41] Néel L. - Ann. Physique, (r 2), i 949, 4, 249.

[42] Chevallier R. et Mathieu Mile S., inédit.

[43] Chevallier R. et Cuny Mile Y., inédit.

[44] Michel A., Chaudron G. et Bénard J. (rapport all présent Congrès).

[45] Pouillard E. - Thèse, Lille, r 9ł.9; Ann. Chim., ı gјo, 16, 164 . 